Supporting information for:

\title{
Isolation and Crystal Structures of Two Singlet Bis(triarylamine) Dications with Non-quinoidal Geometries
}

Shijun Zheng, ${ }^{\dagger, \neq}$ Stephen Barlow, ${ }^{\dagger, \uparrow, \star}$ Chad Risko, ${ }^{\dagger}$ Tiffany L. Kinnibrugh, ${ }^{\S}$ Viktor N. Khrustalev, ${ }^{\Uparrow}$ Simon C. Jones,${ }^{\dagger}$ Mikhail Yu. Antipin,,${ }^{\S \pi}$ Neil M. Tucker, ${ }^{\ddagger}$ Tatiana V. Timofeeva, ${ }^{\S}$ Veaceslav Coropceanu, ${ }^{\dagger}$ Jean-Luc Brédas, ${ }^{\dagger}$ Seth $\mathrm{R}$. Marder $^{\dagger, \ddagger}$

School of Chemistry and Biochemistry and Center for Organic Photonics and Electronics, Georgia Institute of Technology, Atlanta, GA 30332-0400, USA; Department of Chemistry, University of Arizona, Tucson, AZ 85721, USA; Department of Natural Sciences, New Mexico Highlands University, Las Vegas, NM 87701, USA; Institute of Organoelement Compounds, Russian Academy of Sciences, Moscow, Russia

1) Full Citations for References 25, 26 and 42

2) Experimental Details for Physical Measurements and Synthesis of 2

3) ${ }^{1} \mathrm{H}$ and ${ }^{13} \mathrm{C}$ NMR Spectra for III

4) ${ }^{1} \mathrm{H}$ and NOESY NMR Spectra for $[2]^{2+}$

5) Summary of X-Ray Data and Structure of 2

6) Bond Numbering Schemes and Tables of Calculated Geometries and Energies for $[1]^{\mathrm{n}+},[2]^{\mathrm{n}+}$ and the Chichibabin Hydrocarbon

7) Full Coordinates for Calculated Structures

$\dagger$ Georgia Institute of Technology

\$ University of Arizona

$\S$ New Mexico Highlands University

I Russian Academy of Sciences 


\section{Full Citations for References 25, 26 and 42}

(25) Szeghalmi, A. V.; Erdmann, M.; Engel, V.; Schmitt, M.; Amthor, S.; Kriegisch, V.; Nöll, G.; Stahl, R.; Lambert, C.; Leusser, D.; Stalke, D.; Zabel, M.; Popp, J. J. Am. Chem. Soc. 2004, 126, 7834-7845.

(26) Barlow, S.; Risko, C.; Coropceanu, V.; Tucker, N. M.; Jones, S. C.; Levi, Z.; Khrustalev, V. N.; Antipin, M. Y.; Kinnibrugh, T. L.; Timofeeva, T.; Marder, S. R.; Brédas, J. L. Chem. Commun. 2005, 764-766.

(42) Frisch, M. J.; Trucks, G. W.; Schlegel, H. B.; Scuseria, G. E.; Robb, M. A.; Cheeseman, J. R.; Zakrzewski, V. G.; Montgomery, J., J.A.; Stratmann, R. E.; Burant, J. C.; Dapprich, S.; Millam, J. M.; Daniels, A. D.; Kudin, K. N.; Strain, M. C.; Farkas, O.; Tomasi, J.; Barone, V.; Cossi, M.; Cammi, R.; Mennucci, B.; Pomelli, C.; Adamo, C.; Clifford, S.; Ochterski, J.; Petersson, G. A.; Ayala, P. Y.; Cui, Q.; Morokuma, K.; Salvador, P.; Dannenberg, J. J.; Malick, D. K.; Rabuck, A. D.; Raghavachari, K.; Foresman, J. B.; Cioslowski, J.; Ortiz, J. V.; Baboul, A. G.; Stefanov, B. B.; Liu, G.; Liashenko, A.; Piskorz, P.; Komaromi, I.; Gomperts, R.; Martin, R. L.; Fox, D. J.; Keith, T.; Al-Laham, M. A.; Peng, C. Y.; Nanayakkara, A.; Challacombe, M.; Gill, P. M. W.; Johnson, B.; Chen, W.; Wong, M. W.; Andres, J. L.; Gonzalez, C.; Head-Gordon, M.; Replogle, E. S.; Pople, J. A. Gaussian98, Rev. A.11, 1998. 


\section{Experimental Details for Physical Measurements and Synthesis of 2}

Physical Measurements. ESR measurements were conducting using ca. $5 \square 10^{-4} \mathrm{M}$ $\mathrm{CH}_{2} \mathrm{Cl}_{2}$ solutions; $\left[\left(p-{ }^{+} \mathrm{BuC}_{6} \mathrm{H}_{4}\right)_{3} \mathrm{~N}\right]^{+}\left[\mathrm{SbF}_{6}\right]^{-}$, the preparation and characterization of which will be described elsewhere, was used as a standard for estimating spin concentrations. Magnetic measurements were performed using a Quantum Design MPMS-5S SQUID magnetometer in the temperature range $5-350 \mathrm{~K}$ using a $500 \mathrm{G}$ applied field. Electrochemical measurements were carried out under nitrogen on dry deoxygenated dichloromethane solutions ca. $10^{-4} \mathrm{M}$ in analyte and $0.1 \mathrm{M}$ in tetra- $n$-butylammonium hexafluorophosphate using a BAS Potentiostat, a glassy-carbon working electrode, a platinum auxillary electrode, and, as a pseudo-reference electrode, a silver wire anodized in $1 \mathrm{M}$ aqueous potassium chloride. Potentials were referenced to $\left[\mathrm{FeCp}_{2}\right]^{+/ 0}$ by using $\left[\mathrm{CoCp}_{2}\right]^{+}\left[\mathrm{PF}_{6}\right]^{-}\left(-1.32 \mathrm{~V}\right.$ vs. $\left[\mathrm{FeCp}_{2}\right]^{+/ 0}$ in $\left.\mathrm{CH}_{2} \mathrm{Cl}_{2}{ }^{1}\right)$ as an internal reference. Cyclic voltammograms were recorded at a scan rate of $50 \mathrm{mVs}^{-1}$. In addition to synthesizing the dications as isolable salts (described in main text), we also generated the dications of $\mathbf{1}$ and 2 in $\mathrm{CH}_{2} \mathrm{Cl}_{2}$ solution for optical spectroscopic characterization using in situ oxidation with two equivalents of $\left[\left(p-\mathrm{BrC}_{6} \mathrm{H}_{4}\right)_{3} \mathrm{~N}\right]^{+}\left[\mathrm{SbCl}_{6}\right]^{-}\left(E_{1 / 2}=+0.70 \mathrm{~V} \text { vs. } \mathrm{FeCp}_{2}{ }^{+/ 0}\right)^{2} \mathrm{ESR}$ measurements were conducting using a Bruker EMX spectrometer on solutions ca. 5 $10^{-4} \mathrm{M}$ in $\mathrm{CH}_{2} \mathrm{Cl}_{2} ;\left[\left(p-{ }^{-} \mathrm{BuC}_{6} \mathrm{H}_{4}\right)_{3} \mathrm{~N}^{+}\left[\mathrm{SbF}_{6}\right]^{-}\right.$, the preparation and characterization of which will be described elsewhere, was used as a standard for estimating spin concentrations.

(1) Barlow, S. Inorg. Chem. 2001, 40, 7047-7053

(2) Connelly, N. G.; Geiger, W. E. Chem. Rev. 1996, 96, 877-910 


\section{Scheme S1}

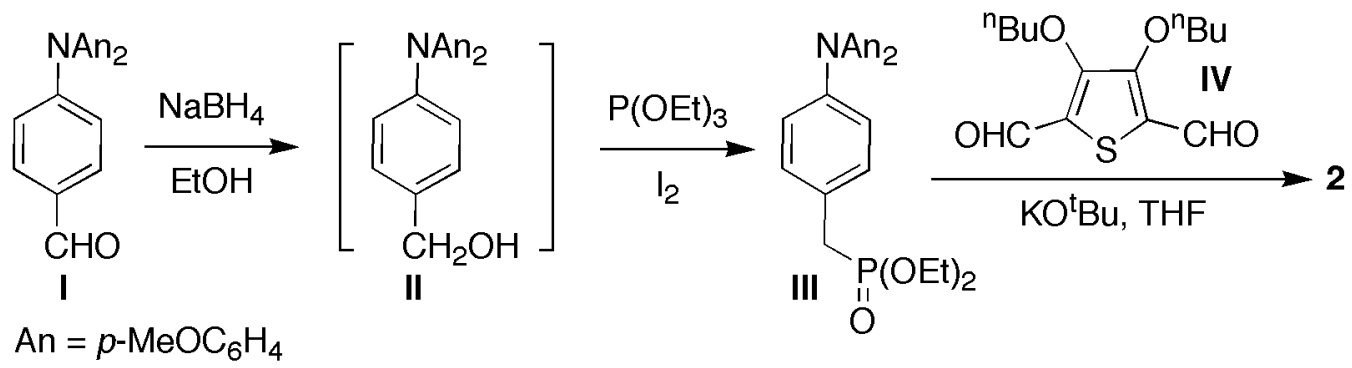

III. To a solution of $\mathbf{I}^{3}(8.50 \mathrm{~g}, 25 \mathrm{mmol})$ in ethanol $(500 \mathrm{~mL})$ was added sodium borohydride $(1.9 \mathrm{~g}, 51 \mathrm{mmol})$. The mixture was stirred at room temperature for $4 \mathrm{~h}$. The solvent was concentrated under reduced pressure and the reaction mixture was worked up with saturated aqueous $\mathrm{NH}_{4} \mathrm{Cl}$ solution and chloroform $(3 \square 200 \mathrm{~mL})$. The organic phase was dried over $\mathrm{MgSO}_{4}$; removal of solvent under reduced pressure gave the alcohol, II, as a yellow viscous liquid (8.0g). Since the alcohol underwent ether formation on storage, it was used without further purification in the next reaction, which was performed according to a general procedure described elsewhere ${ }^{4}$ using crude II $(8.0 \mathrm{~g})$, triethyl phosphite $(50 \mathrm{~mL})$, and iodine $(6.1 \mathrm{~g}, 24 \mathrm{mmol})$, with a reaction time of $15 \mathrm{~h}$ at a temperature of $0{ }^{\circ} \mathrm{C}$. After removal of the excess triethyl phosphite under reduced pressure, the resulting residue was purified by column chromatography (silica, first using dichloromethane, then using ethyl acetate as eluent) to give compound III as a pale yellow viscous liquid $\left(9.8 \mathrm{~g}, 22 \mathrm{mmol}, 86 \%\right.$ from I). ${ }^{1} \mathrm{H}$ NMR $\left(400 \mathrm{MHz}, \mathrm{CD}_{2} \mathrm{Cl}_{2}\right) \square 7.07$ (dd, $J=2.5,8.7 \mathrm{~Hz}, 2 \mathrm{H}), 7.01(\mathrm{~d}, J=9.0 \mathrm{~Hz}, 4 \mathrm{H}), 6.83(\mathrm{~m}, 6 \mathrm{H}), 4.00(\mathrm{~m}, 4 \mathrm{H}), 3.77(\mathrm{~s}$, $6 \mathrm{H}), 3.03(\mathrm{~d}, J=21.2 \mathrm{~Hz}, 2 \mathrm{H}), 1.23(\mathrm{t}, J=7.0 \mathrm{~Hz}, 6 \mathrm{H}) .{ }^{13} \mathrm{C} \mathrm{NMR}\left(100 \mathrm{MHz}, \mathrm{CD}_{2} \mathrm{Cl}_{2}\right) \square$

(3) I has previously been obtained by a variety of syntheses; we obtained it in $79 \%$ yield through the Vilsmeier formylation of $N$, $N$-bis(4methoxyphenyl)-N-phenylamine, which was itself obtained using Pd-catalyzed coupling chemistry from bis(4-methoxyphenyl)amine and bromobenzene.

(4) Zheng, S. J.; Barlow, S.; Parker, T. C.; Marder, S. R. Tetrahedron Lett. 2003, 44, 7989-7992 
$156.3,148.0\left(\mathrm{~d}, J_{\mathrm{CP}}=3.2 \mathrm{~Hz}\right), 141.3,130.6\left(\mathrm{~d}, J_{\mathrm{CP}}=6.5 \mathrm{~Hz}\right), 126.8,123.9\left(\mathrm{~d}, J_{\mathrm{CP}}=9.3\right.$ $\mathrm{Hz}), 120.9\left(\mathrm{~d}, J_{\mathrm{CP}}=2.8 \mathrm{~Hz}\right), 114.9,62.3\left(\mathrm{~d}, J_{\mathrm{CP}}=6.5 \mathrm{~Hz}\right), 55.8,33.0\left(\mathrm{~d}, J_{\mathrm{CP}}=138.3 \mathrm{~Hz}\right)$, $16.6\left(\mathrm{~d}, J_{\mathrm{CP}}=5.9 \mathrm{~Hz}\right)$. See section 3 for NMR spectra. HRMS (EI) calcd for $\mathrm{C}_{25} \mathrm{H}_{30} \mathrm{NO}_{5} \mathrm{P}$ : 455.1862. Found: 455.1822.

2. To a solution of III $(0.65 \mathrm{~g}, 1.4 \mathrm{mmol})$ and $\mathbf{I V}^{5}(0.203 \mathrm{~g}, 0.72 \mathrm{mmol})$ in THF $(15 \mathrm{~mL})$, was added potassium tert-butoxide $(0.36 \mathrm{~g}, 3.2 \mathrm{mmol})$ at $-78{ }^{\circ} \mathrm{C}$ under nitrogen. The mixture was stirred at $-78{ }^{\circ} \mathrm{C}$ and warmed slowly to room temperature. After removal of solvent, the residue was worked up with saturated aqueous $\mathrm{NH}_{4} \mathrm{Cl}$ solution and dichloromethane (3 $\square 50 \mathrm{~mL}$ ). After the organic phase was dried over $\mathrm{MgSO}_{4}$ and the solvent was removed under reduced pressure, column chromatography (silica, 2:1 dichloromethane / hexanes) gave the product as orange solids $(0.45 \mathrm{~g}, 70 \%)$. Single crystals of 2 were obtained by slow evaporation of a solution in dichloromethane / methanol. ${ }^{1} \mathrm{H}$ NMR (400 MHz, $\left.\mathrm{CD}_{2} \mathrm{Cl}_{2}\right) \square 7.29(\mathrm{~d}, J=8.8 \mathrm{~Hz}, 4 \mathrm{H}), 7.10(\mathrm{~d}, J=16.2 \mathrm{~Hz}$, 2H), $7.08(\mathrm{~d}, J=9.0 \mathrm{~Hz}, 8 \mathrm{H}), 6.87(\mathrm{~d}, J=8.7 \mathrm{~Hz}, 4 \mathrm{H}), 6.85(\mathrm{~d}, J=9.0 \mathrm{~Hz}, 8 \mathrm{H}), 6.75(\mathrm{~d}$, $J=16.1 \mathrm{~Hz}, 2 \mathrm{H}), 4.07(\mathrm{t}, J=6.6 \mathrm{~Hz}, 4 \mathrm{H}), 3.79(\mathrm{~s}, 12 \mathrm{H}), 1.76(\mathrm{~m}, 4 \mathrm{H}), 1.55(\mathrm{~m}, 2 \mathrm{H}), 1.00$ (t, $J=7.4 \mathrm{~Hz}, 6 \mathrm{H}) .{ }^{13} \mathrm{C}$ NMR $\left(100 \mathrm{MHz}, \mathrm{CD}_{2} \mathrm{Cl}_{2}\right) \square 156.6,148.6,147.5,140.9,129.6$, 127.2, 127.1, 126.2, 123.9, 120.4, 116.1, 115.0, 74.0, 55.8, 32.5, 19.6, 14.1. HRMS (FAB) calcd for $\mathrm{C}_{56} \mathrm{H}_{58} \mathrm{~N}_{2} \mathrm{O}_{6} \mathrm{~S}:$ 886.4016. Found: 886.3998. Anal. calcd for $\mathrm{C}_{56} \mathrm{H}_{58} \mathrm{~N}_{2} \mathrm{O}_{6} \mathrm{~S}$ : C, 75.82; H, 6.59; N, 3.16. Found: C, 75.81; H, 6.60; N, 3.14. UV-vis $\left(\mathrm{CH}_{2} \mathrm{Cl}_{2}\right) \square_{\max }\left(\square_{\max }\right)$ $453(68500) \mathrm{nm}\left(\mathrm{M}^{-1} \mathrm{~cm}^{-1}\right)$.

(5) Ono, N.; Okumura, H.; Murashima, T. Heteroatom Chem. 2001, 12, 414-417 
3. ${ }^{1} \mathrm{H}$ and ${ }^{13} \mathrm{C}$ NMR Spectra for III

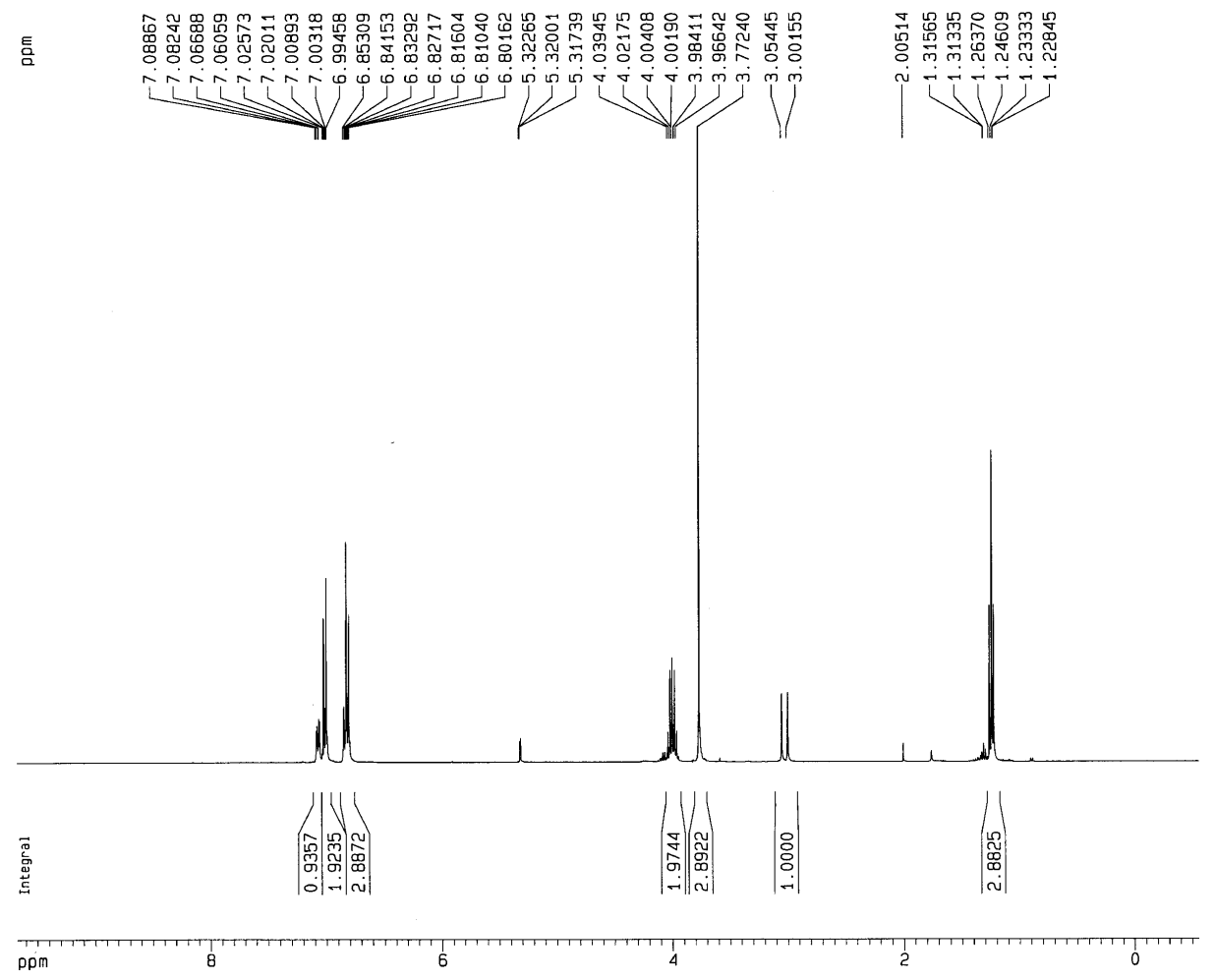

Figure S1. ${ }^{1} \mathrm{H}$ NMR spectrum of III in $\mathrm{CD}_{2} \mathrm{Cl}_{2}$.

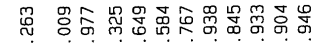

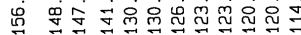

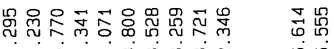

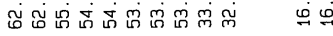

i

VYWV

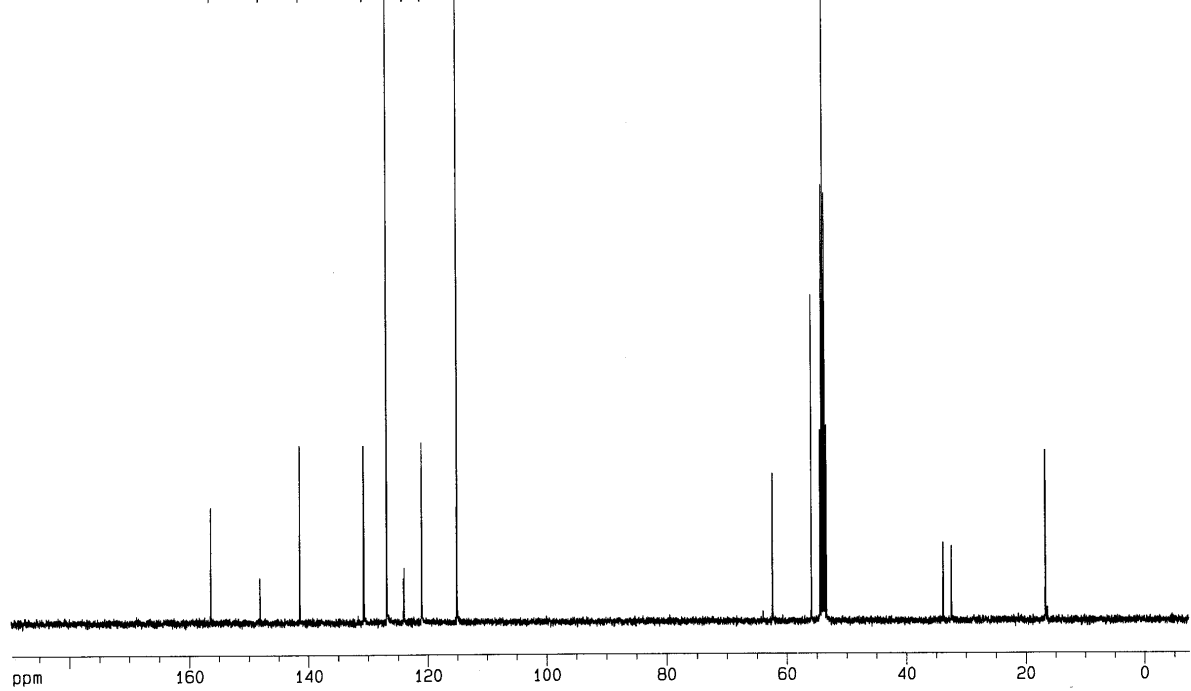

Figure S2. ${ }^{13} \mathrm{C}\left\{{ }^{1} \mathrm{H}\right\}$ NMR spectrum of III in $\mathrm{CD}_{2} \mathrm{Cl}_{2}$. 


\section{4. ${ }^{1} \mathrm{H}$ and NOESY NMR Spectra for $[2]^{2+}$}

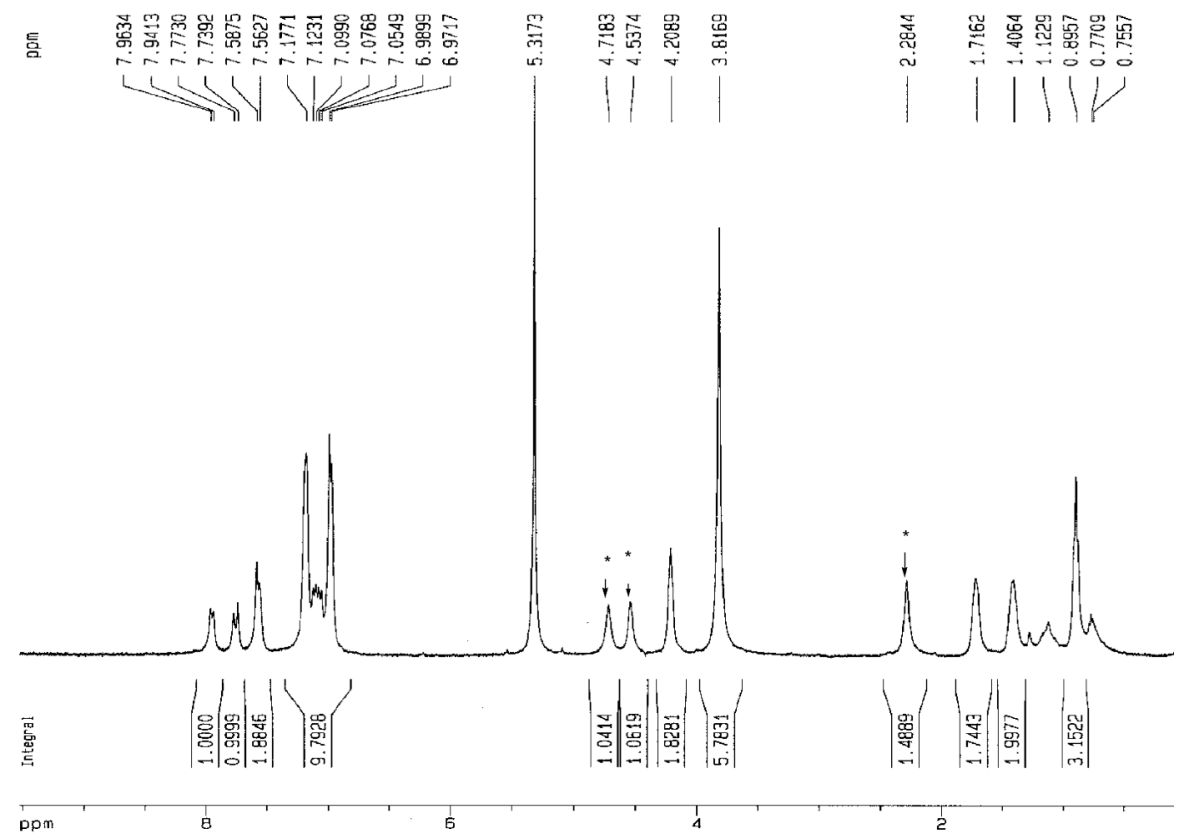

Figure S3. ${ }^{1} \mathrm{H}$ NMR spectrum of the dication of $[2]^{2+}\left(\left[\mathrm{SbF}_{6}\right]^{-}\right)_{2}$ at $-85^{\circ} \mathrm{C}$ in $\mathrm{CD}_{2} \mathrm{Cl}_{2}$ (features marked “*” are attributable to $\left.\mathrm{Fe}\left(\mathrm{C}_{5} \mathrm{H}_{4} \mathrm{Ac}\right)_{2}\right)$.

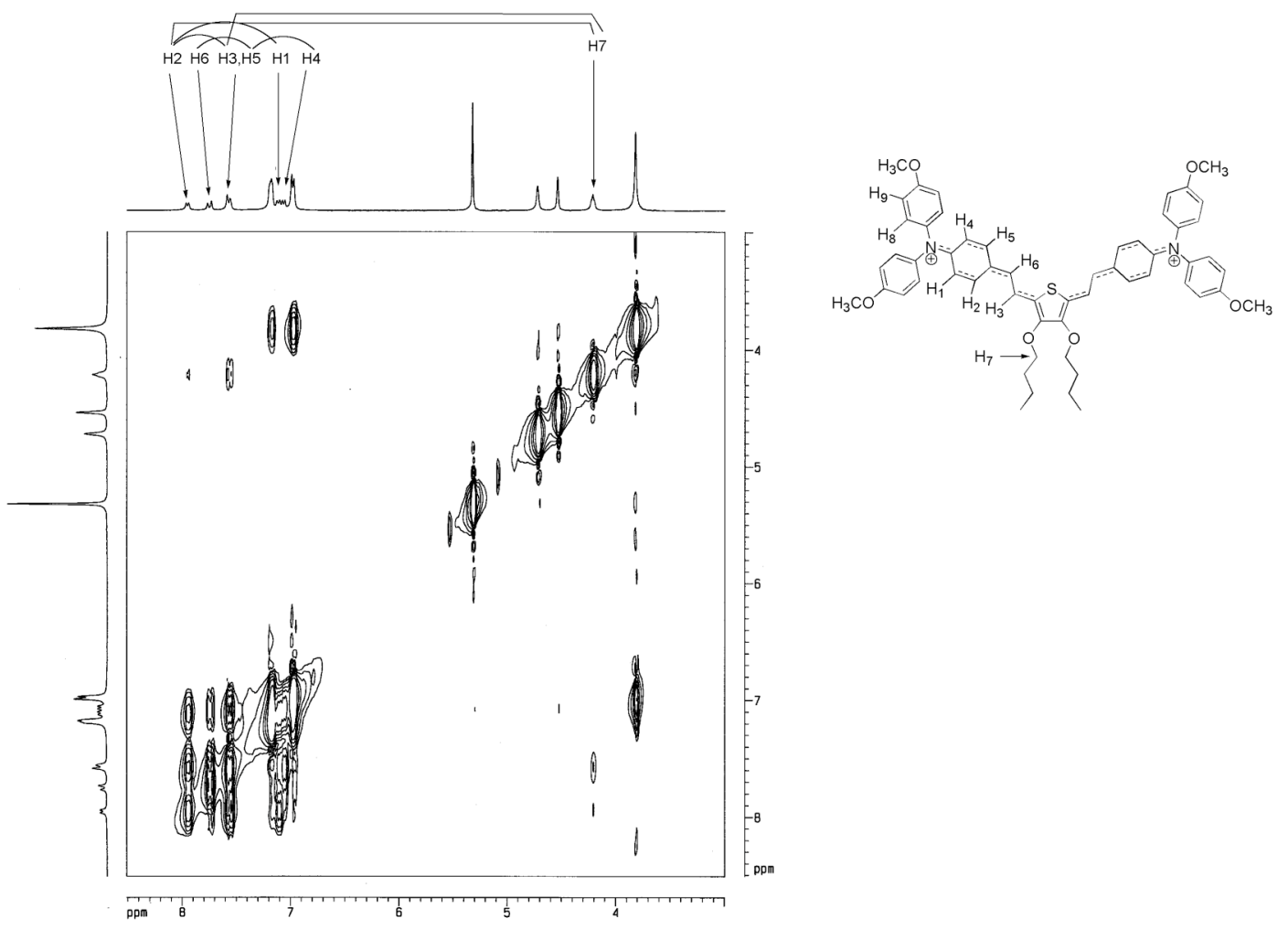

Figure S4. NOESY NMR spectrum of $[2]^{2+}\left(\left[\mathrm{SbF}_{6}\right]^{-}\right)_{2}$ at $-85^{\circ} \mathrm{C}$ in $\mathrm{CD}_{2} \mathrm{Cl}_{2}$. 


\section{Summary of X-Ray Data and Structure of 2}

Table S1. Crystallographic data for $[1]^{2+}\left(\left[\mathrm{SbCl}_{6}\right]^{-}\right)_{2}, 2$ and $[2]^{2+}\left(\left[\mathrm{SbF}_{6}\right]^{-}\right)_{2}$.

\begin{tabular}{|c|c|c|c|}
\hline & {$[\mathbf{1}]^{2+}\left(\left[\mathrm{SbCl}_{6}\right]^{-}\right)_{2}$} & 2 & {$[2]^{2+}\left(\left[\mathrm{SbF}_{6}\right]^{-}\right)_{2}$} \\
\hline Empirical formula & $\mathrm{C}_{42} \mathrm{H}_{38} \mathrm{Cl}_{12} \mathrm{~N}_{2} \mathrm{O}_{4} \mathrm{Sb}_{2}$ & $\mathrm{C}_{56} \mathrm{H}_{58} \mathrm{~N}_{2} \mathrm{O}_{6} \mathrm{~S}$ & $\mathrm{C}_{56} \mathrm{H}_{58} \mathrm{~F}_{12} \mathrm{~N}_{2} \mathrm{O}_{6} \mathrm{SSb}_{2}$ \\
\hline Formula weight & 1303.64 & 887.10 & 1358.60 \\
\hline Crystal system & Monoclinic & Triclinic & Orthorhombic \\
\hline Space group & $P 2_{1} / n$ & $P-1$ & $P 2_{1} 2_{1} 2_{1}$ \\
\hline$a / \AA$ & $12.0382(9)$ & $11.6716(9)$ & $13.0866(17)$ \\
\hline$b / \AA$ & $14.4404(11)$ & $14.9373(11)$ & $14.3660(19)$ \\
\hline$c / \AA$ & $15.2760(12)$ & $15.5890(11)$ & $30.542(4)$ \\
\hline$\square /{ }^{\circ}$ & 90 & $64.936(2)$ & 90 \\
\hline$\square / 1^{\circ}$ & $109.749(2)$ & $83.166(2)$ & 90 \\
\hline$\square /{ }^{\circ}$ & 90 & $76.083(2)$ & 90 \\
\hline Volume / $\AA^{3}$ & $2499.3(3)$ & $2389.2(3)$ & $5741.9(13)$ \\
\hline$Z$ & 2 & 2 & 4 \\
\hline$\square($ calcd $) / \mathrm{Mg} \cdot \mathrm{m}^{-3}$ & 1.732 & 1.233 & 1.572 \\
\hline$\square / \mathrm{mm}^{-1}$ & 1.765 & 0.121 & 1.066 \\
\hline $\mathrm{F}(000)$ & 1284 & 944 & 2728 \\
\hline Crystal size $/ \mathrm{mm}^{3}$ & $0.30 \square 0.24 \square 0.21$ & $0.21 \square 0.18 \square 0.08$ & $0.40 \square 0.20 \square 0.10$ \\
\hline$\square$ range for data $/^{\circ}$ & $1.88-26.02$ & $1.80-25.06$ & $1.69-25.06$ \\
\hline \multirow[t]{3}{*}{ Index ranges } & $-14 \square h \square 14$ & $-13 \square h \square 13$ & $-15 \square h \square 15$ \\
\hline & $-17 \square k \square 17$ & $-17 \square k \square 17$ & $-17 \square k \square 17$ \\
\hline & 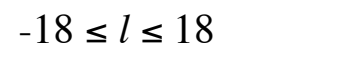 & $-18 \square l \square 18$ & $-29 \square l \square 36$ \\
\hline Reflns collected & 21899 & 19776 & 36672 \\
\hline Indept reflns $\left(R_{\text {int }}\right)$ & $4880(0.0211)$ & $8409(0.0544)$ & $10068(0.0848)$ \\
\hline $\begin{array}{l}\text { Max. and min. } \\
\text { transmission }\end{array}$ & 0.696 and 0.606 & 0.990 and 0.975 & 0.901 and 0.675 \\
\hline Data/restraints/params & 4897 / 18 / 352 & $8409 / 0 / 818$ & $10068 / 18 / 726$ \\
\hline Goodness-of-fit on $F^{2}$ & 1.007 & 1.039 & 1.035 \\
\hline Final $R 1, w R 2[I>2 \square(I)]$ & $0.0414,0.0975$ & $0.0513,0.0821$ & $0.0575,0.1102$ \\
\hline Final $R 1, w R 2$ (all data) & $0.0500,0.1034$ & $0.1068,0.0938$ & $0.1002,0.1187$ \\
\hline $\begin{array}{l}\text { Largest peak and hole / } \\
\mathrm{e}^{-3}\end{array}$ & 0.977 and -1.160 & 0.219 and -0.258 & 1.697 and -0.527 \\
\hline
\end{tabular}

Further details are supplied as CIF files. 


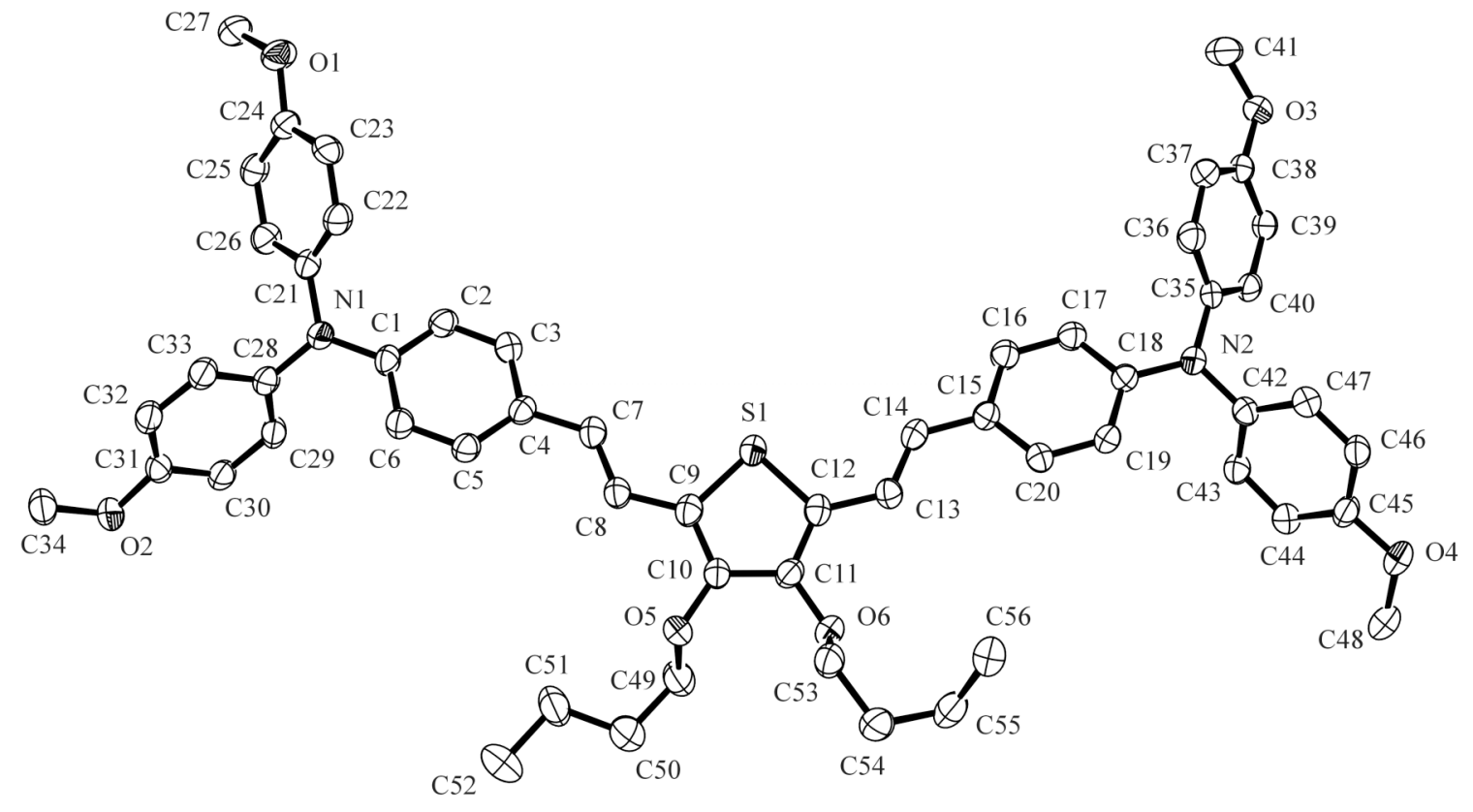

Figure S5. Crystal structure of 2. 
6. Bond Numbering Schemes and Tables of Calculated Geometries and Energies for $[1]^{\mathrm{n}+},[2]^{\mathrm{n}+}$ and the Chichibabin Hydrocarbon

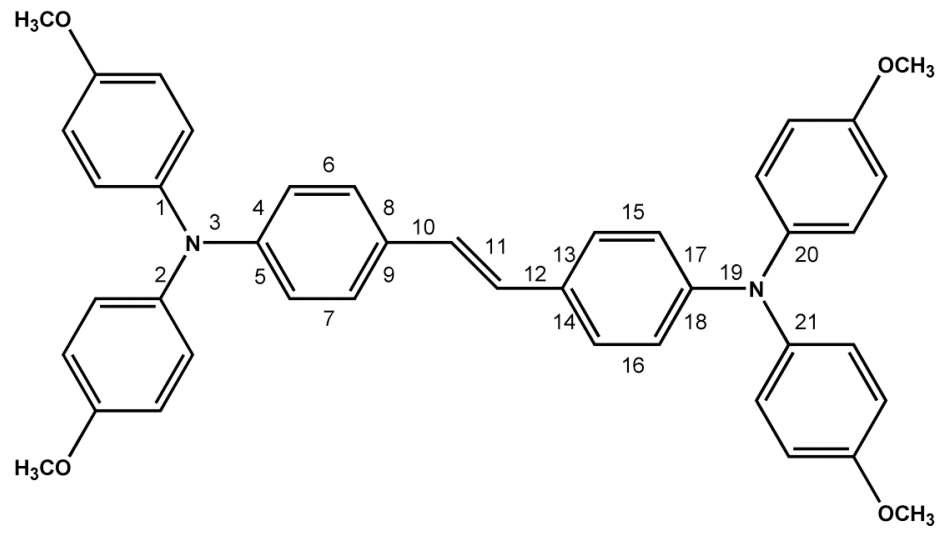

Figure S6. Bond numbering scheme used for calculations on $[\mathbf{1}]^{\text {n+ }}$

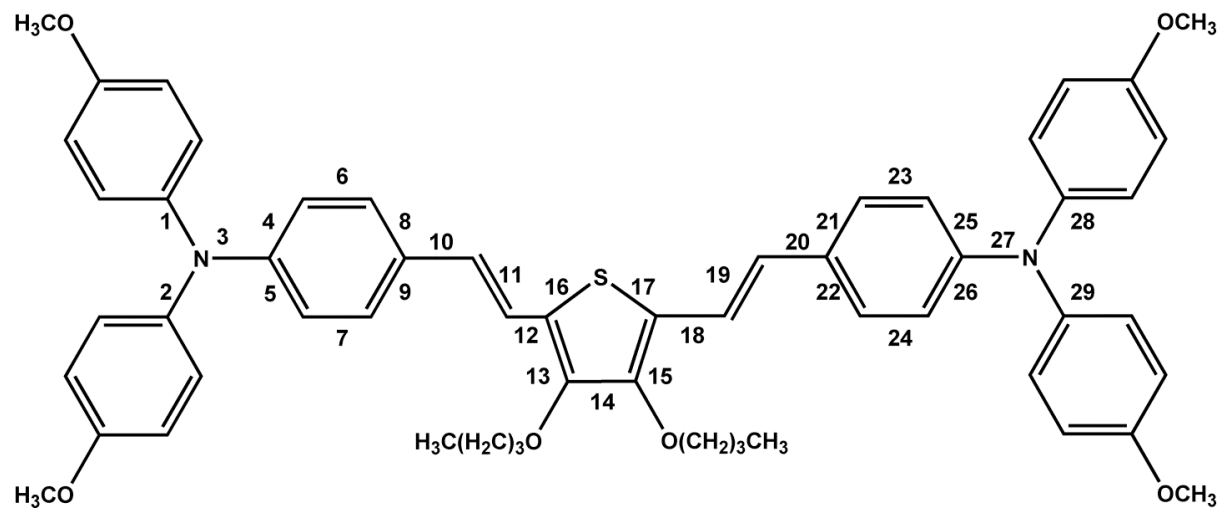

Figure S7. Bond numbering scheme for $[2]^{\text {n+ }}$.

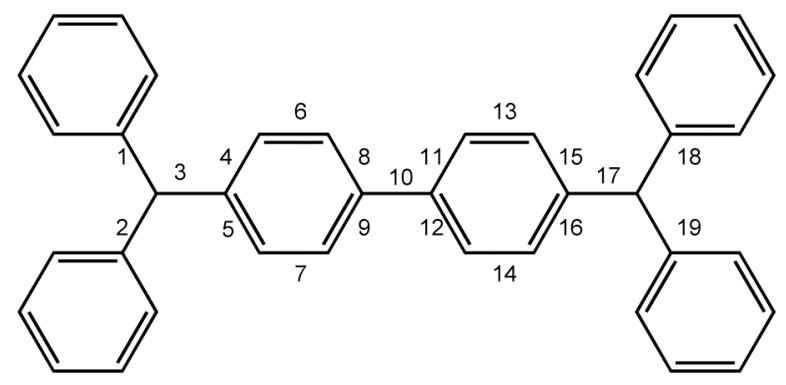

Figure S8. Bond numbering scheme for Chichibabin's hydrocarbon. 
Table S2. Selected bond lengths $(\AA)$ for $[1]^{\mathrm{n}+}$ at the B3LYP/6-31G* level.

\begin{tabular}{lccccc}
\hline Bond no. & Neutral & Cation & \multicolumn{3}{c}{ Dication } \\
& & & Singlet & BS & Triplet \\
\hline & & & & & \\
2 & 1.425 & 1.429 & 1.422 & 1.413 & 1.409 \\
3 & 1.425 & 1.429 & 1.423 & 1.414 & 1.410 \\
4 & 1.413 & 1.388 & 1.379 & 1.407 & 1.419 \\
5 & 1.406 & 1.418 & 1.425 & 1.411 & 1.406 \\
6 & 1.410 & 1.421 & 1.428 & 1.414 & 1.409 \\
7 & 1.390 & 1.378 & 1.372 & 1.383 & 1.387 \\
8 & 1.386 & 1.377 & 1.371 & 1.381 & 1.385 \\
9 & 1.408 & 1.419 & 1.428 & 1.416 & 1.412 \\
10 & 1.411 & 1.421 & 1.429 & 1.417 & 1.413 \\
11 & 1.461 & 1.436 & 1.420 & 1.450 & 1.460 \\
12 & 1.352 & 1.370 & 1.385 & 1.362 & 1.354 \\
13 & 1.461 & 1.436 & 1.420 & 1.450 & 1.460 \\
14 & 1.411 & 1.421 & 1.429 & 1.417 & 1.413 \\
15 & 1.408 & 1.419 & 1.428 & 1.416 & 1.412 \\
16 & 1.386 & 1.377 & 1.371 & 1.381 & 1.385 \\
17 & 1.390 & 1.378 & 1.372 & 1.383 & 1.387 \\
18 & 1.410 & 1.421 & 1.428 & 1.414 & 1.409 \\
19 & 1.406 & 1.418 & 1.425 & 1.411 & 1.406 \\
20 & 1.413 & 1.388 & 1.379 & 1.407 & 1.419 \\
21 & 1.425 & 1.429 & 1.423 & 1.414 & 1.410 \\
\hline & 1.425 & 1.429 & 1.422 & 1.413 & 1.409 \\
\hline & & & & & \\
\hline
\end{tabular}


Table S3. Selected geometric parameters $(\AA)$ for $[2]^{\mathrm{n}+}$ at the B3LYP/6-31G* level.

\begin{tabular}{|c|c|c|c|c|c|}
\hline \multirow[t]{2}{*}{ Bond no. } & \multirow[t]{2}{*}{ Neutral } & \multirow[t]{2}{*}{ Cation } & \multicolumn{3}{|c|}{ Dication } \\
\hline & & & Singlet & $\mathrm{BS}$ & Triplet \\
\hline 1 & 1.425 & 1.432 & 1.430 & 1.422 & 1.417 \\
\hline 2 & 1.425 & 1.432 & 1.430 & 1.422 & 1.416 \\
\hline 3 & 1.412 & 1.387 & 1.373 & 1.391 & 1.405 \\
\hline 4 & 1.410 & 1.421 & 1.429 & 1.421 & 1.415 \\
\hline 5 & 1.406 & 1.418 & 1.426 & 1.418 & 1.412 \\
\hline 6 & 1.386 & 1.377 & 1.370 & 1.376 & 1.380 \\
\hline 7 & 1.389 & 1.379 & 1.371 & 1.377 & 1.382 \\
\hline 8 & 1.411 & 1.421 & 1.430 & 1.423 & 1.418 \\
\hline 9 & 1.409 & 1.419 & 1.428 & 1.421 & 1.417 \\
\hline 10 & 1.457 & 1.432 & 1.414 & 1.432 & 1.445 \\
\hline 11 & 1.356 & 1.375 & 1.391 & 1.375 & 1.365 \\
\hline 12 & 1.437 & 1.413 & 1.397 & 1.414 & 1.426 \\
\hline 13 & 1.385 & 1.409 & 1.423 & 1.408 & 1.398 \\
\hline 14 & 1.427 & 1.409 & 1.397 & 1.412 & 1.423 \\
\hline 15 & 1.385 & 1.407 & 1.435 & 1.411 & 1.397 \\
\hline 16 & 1.760 & 1.761 & 1.764 & 1.759 & 1.757 \\
\hline 17 & 1.760 & 1.761 & 1.762 & 1.759 & 1.756 \\
\hline 18 & 1.437 & 1.414 & 1.391 & 1.412 & 1.427 \\
\hline 19 & 1.356 & 1.374 & 1.395 & 1.376 & 1.364 \\
\hline 20 & 1.457 & 1.433 & 1.410 & 1.431 & 1.446 \\
\hline 21 & 1.411 & 1.420 & 1.431 & 1.423 & 1.418 \\
\hline 22 & 1.409 & 1.418 & 1.430 & 1.422 & 1.416 \\
\hline 23 & 1.386 & 1.377 & 1.369 & 1.375 & 1.380 \\
\hline 24 & 1.389 & 1.379 & 1.370 & 1.377 & 1.382 \\
\hline 25 & 1.410 & 1.421 & 1.431 & 1.422 & 1.415 \\
\hline 26 & 1.406 & 1.418 & 1.428 & 1.419 & 1.412 \\
\hline 27 & 1.411 & 1.387 & 1.371 & 1.390 & 1.405 \\
\hline 28 & 1.425 & 1.432 & 1.430 & 1.422 & 1.416 \\
\hline 29 & 1.425 & 1.432 & 1.430 & 1.423 & 1.416 \\
\hline
\end{tabular}


Table S4. Selected bond lengths ( $\AA$ ) for the closed-shell singlet, BS (biradical), and triplet configurations of Chichibabin's hydrocarbon at the B3LYP/6-31G* level.

\begin{tabular}{lccc}
\hline Bond number & Singlet & BS & Triplet \\
\hline 1 & & & \\
2 & 1.479 & 1.471 & 1.465 \\
3 & 1.479 & 1.471 & 1.465 \\
4 & 1.402 & 1.442 & 1.463 \\
5 & 1.445 & 1.425 & 1.415 \\
6 & 1.445 & 1.425 & 1.415 \\
7 & 1.365 & 1.381 & 1.388 \\
8 & 1.365 & 1.381 & 1.388 \\
9 & 1.437 & 1.416 & 1.408 \\
10 & 1.437 & 1.416 & 1.408 \\
11 & 1.420 & 1.463 & 1.480 \\
12 & 1.437 & 1.416 & 1.408 \\
13 & 1.437 & 1.416 & 1.408 \\
14 & 1.365 & 1.138 & 1.388 \\
15 & 1.365 & 1.381 & 1.388 \\
16 & 1.445 & 1.425 & 1.415 \\
17 & 1.445 & 1.425 & 1.415 \\
18 & 1.402 & 1.442 & 1.463 \\
19 & 1.479 & 1.471 & 1.465 \\
& 1.479 & 1.471 & 1.465 \\
\hline
\end{tabular}

Table S5. Comparison of Bond Lengths ( $\AA$ ) and $\mathrm{C}_{6} \mathrm{H}_{4}-\mathrm{C}_{6} \mathrm{H}_{4}$ dihedral $\left({ }^{\circ}\right)$ for Chichibabin's hydrocarbon from X-ray and from DFT for closed-shell-singlet, BS (biradical), and triplet configurations.

\begin{tabular}{llllll}
\hline & $\mathrm{Ph}-\mathrm{C}$ & $\mathrm{C}-\mathrm{C}_{6} \mathrm{H}_{4}$ & $\mathrm{BLA}\left(\mathrm{C}_{6} \mathrm{H}_{4}\right)^{\mathrm{b}}$ & $\mathrm{C}_{6} \mathrm{H}_{4}-\mathrm{C}_{6} \mathrm{H}_{4}$ & dihedral \\
\hline X-ray $^{[3]}$ & $1.470(13)$ & $1.415(3)$ & $0.052(2)$ & $1.448(4)$ & 0.6 \\
Singlet & 1.479 & 1.402 & 0.076 & 1.420 & 9.8 \\
$B S$ & 1.471 & 1.422 & 0.040 & 1.463 & 24.3 \\
Triplet & 1.465 & 1.463 & 0.024 & 1.480 & 34.6 \\
\hline
\end{tabular}


Table S6. Optimized energies (hartrees) and energy splittings (vs. BS, eV) for $[1]^{2+},[2]^{2+}$, and Chichibabin's hydrocarbon at the B3LYP/6-31G* level.

\begin{tabular}{llcc}
\hline & \multicolumn{1}{c}{$\begin{array}{c}\text { Energy } \\
(\mathrm{hr})\end{array}$} & $\begin{array}{c}\text { Splitting } \\
(\mathrm{eV})\end{array}$ \\
\hline$[\mathbf{1 1}]^{2+}$ & Singlet & -2033.22342184 & 0.17 \\
& BS & -2033.22949390 & 0.00 \\
& Triplet & -2033.22741720 & 0.06 \\
\hline$[\mathbf{2}]^{2+}$ & Singlet & -3127.40170528 & 0.07 \\
& BS & -3127.40424931 & 0.00 \\
& Triplet & -3127.40073782 & 0.10 \\
Ch. hydro. & Singlet & -1464.87576091 & 0.14 \\
& BS & -1464.88095919 & 0.00 \\
& Triplet & -1464.87753662 & 0.09 \\
\hline
\end{tabular}




\section{Full Coordinates for Calculated Structures}

Table S7. XYZ coordinates and energies (hr) for $\mathbf{1}$ and $\mathbf{1}^{+}$.

\begin{tabular}{|c|c|c|c|c|c|c|c|}
\hline \multicolumn{4}{|c|}{$\begin{array}{l}\mathbf{1} \\
(-2033.69561670 \mathrm{hr})\end{array}$} & \multicolumn{4}{|c|}{$\begin{array}{l}\mathbf{1}^{+} \\
(-2033.50753780 \mathrm{hr})\end{array}$} \\
\hline $\mathrm{N}$ & 2.165 & -5.683 & 1.071 & $\mathrm{~N}$ & 2.174 & -5.631 & 1.066 \\
\hline $\mathrm{O}$ & 3.512 & -8.835 & -3.365 & $\mathrm{O}$ & 3.449 & -8.850 & -3.318 \\
\hline $\mathrm{O}$ & 2.641 & -7.952 & 6.176 & $\mathrm{O}$ & 2.679 & -7.970 & 6.115 \\
\hline $\mathrm{C}$ & 0.357 & -0.280 & 0.501 & $\mathrm{C}$ & 0.317 & -0.294 & 0.531 \\
\hline $\mathrm{C}$ & 0.794 & -1.669 & 0.609 & $\mathrm{C}$ & 0.768 & -1.654 & 0.623 \\
\hline $\mathrm{C}$ & 1.569 & -2.064 & 1.716 & $\mathrm{C}$ & 1.437 & -2.083 & 1.799 \\
\hline $\mathrm{C}$ & 2.028 & -3.366 & 1.873 & $\mathrm{C}$ & 1.896 & -3.373 & 1.955 \\
\hline $\mathrm{C}$ & 1.714 & -4.352 & 0.923 & $\mathrm{C}$ & 1.717 & -4.329 & 0.924 \\
\hline $\mathrm{C}$ & 0.927 & -3.976 & -0.184 & $\mathrm{C}$ & 1.049 & -3.915 & -0.261 \\
\hline $\mathrm{C}$ & 0.488 & -2.670 & -0.336 & $\mathrm{C}$ & 0.590 & -2.625 & -0.399 \\
\hline $\mathrm{C}$ & 2.521 & -6.457 & -0.071 & $\mathrm{C}$ & 2.507 & -6.425 & -0.075 \\
\hline $\mathrm{C}$ & 3.327 & -5.924 & -1.082 & $\mathrm{C}$ & 3.348 & -5.922 & -1.075 \\
\hline $\mathrm{C}$ & 3.671 & -6.680 & -2.205 & $\mathrm{C}$ & 3.684 & -6.699 & -2.182 \\
\hline $\mathrm{C}$ & 3.228 & -8.002 & -2.319 & $\mathrm{C}$ & 3.186 & -8.006 & -2.293 \\
\hline $\mathrm{C}$ & 2.430 & -8.549 & -1.302 & $\mathrm{C}$ & 2.349 & -8.516 & -1.283 \\
\hline $\mathrm{C}$ & 2.074 & -7.784 & -0.200 & $\mathrm{C}$ & 2.014 & -7.737 & -0.189 \\
\hline $\mathrm{C}$ & 2.317 & -6.256 & 2.366 & $\mathrm{C}$ & 2.333 & -6.220 & 2.358 \\
\hline $\mathrm{C}$ & 1.298 & -6.141 & 3.329 & $\mathrm{C}$ & 1.297 & -6.152 & 3.309 \\
\hline $\mathrm{C}$ & 1.443 & -6.706 & 4.588 & $\mathrm{C}$ & 1.449 & -6.742 & 4.552 \\
\hline $\mathrm{C}$ & 2.603 & -7.425 & 4.915 & $\mathrm{C}$ & 2.635 & -7.426 & 4.876 \\
\hline $\mathrm{C}$ & 3.618 & -7.558 & 3.962 & $\mathrm{C}$ & 3.666 & -7.506 & 3.927 \\
\hline $\mathrm{C}$ & 3.474 & -6.966 & 2.705 & $\mathrm{C}$ & 3.510 & -6.904 & 2.681 \\
\hline $\mathrm{C}$ & 3.791 & -8.688 & 6.558 & $\mathrm{C}$ & 3.843 & -8.693 & 6.508 \\
\hline $\mathrm{C}$ & 4.318 & -8.332 & -4.418 & $\mathrm{C}$ & 4.292 & -8.404 & -4.376 \\
\hline $\mathrm{C}$ & -0.357 & 0.280 & -0.501 & $\mathrm{C}$ & -0.317 & 0.294 & -0.531 \\
\hline $\mathrm{N}$ & -2.165 & 5.683 & -1.071 & $\mathrm{~N}$ & -2.174 & 5.631 & -1.066 \\
\hline $\mathrm{O}$ & -3.512 & 8.835 & 3.365 & $\mathrm{O}$ & -3.449 & 8.850 & 3.318 \\
\hline $\mathrm{O}$ & -2.641 & 7.952 & -6.176 & $\mathrm{O}$ & -2.679 & 7.970 & -6.115 \\
\hline $\mathrm{C}$ & -0.794 & 1.669 & -0.609 & $\mathrm{C}$ & -0.768 & 1.654 & -0.623 \\
\hline $\mathrm{C}$ & -1.569 & 2.064 & -1.716 & $\mathrm{C}$ & -1.437 & 2.083 & -1.799 \\
\hline $\mathrm{C}$ & -2.028 & 3.366 & -1.873 & $\mathrm{C}$ & -1.896 & 3.373 & -1.955 \\
\hline $\mathrm{C}$ & -1.714 & 4.352 & -0.923 & $\mathrm{C}$ & -1.717 & 4.329 & -0.924 \\
\hline $\mathrm{C}$ & -0.927 & 3.976 & 0.184 & $\mathrm{C}$ & -1.049 & 3.915 & 0.261 \\
\hline $\mathrm{C}$ & -0.488 & 2.670 & 0.336 & $\mathrm{C}$ & -0.590 & 2.625 & 0.399 \\
\hline $\mathrm{C}$ & -2.521 & 6.457 & 0.071 & $\mathrm{C}$ & -2.507 & 6.425 & 0.075 \\
\hline $\mathrm{C}$ & -3.327 & 5.924 & 1.082 & $\mathrm{C}$ & -3.348 & 5.922 & 1.075 \\
\hline $\mathrm{C}$ & -3.671 & 6.680 & 2.205 & $\mathrm{C}$ & -3.684 & 6.699 & 2.182 \\
\hline $\mathrm{C}$ & -3.228 & 8.002 & 2.319 & $\mathrm{C}$ & -3.186 & 8.006 & 2.293 \\
\hline $\mathrm{C}$ & -2.430 & 8.549 & 1.302 & $\mathrm{C}$ & -2.349 & 8.516 & 1.283 \\
\hline $\mathrm{C}$ & -2.074 & 7.784 & 0.200 & $\mathrm{C}$ & -2.014 & 7.737 & 0.189 \\
\hline $\mathrm{C}$ & -2.317 & 6.256 & -2.366 & $\mathrm{C}$ & -2.333 & 6.220 & -2.358 \\
\hline $\mathrm{C}$ & -1.298 & 6.141 & -3.329 & $\mathrm{C}$ & -1.297 & 6.152 & -3.309 \\
\hline $\mathrm{C}$ & -1.443 & 6.706 & -4.588 & $\mathrm{C}$ & -1.449 & 6.742 & -4.552 \\
\hline $\mathrm{C}$ & -2.603 & 7.425 & -4.915 & $\mathrm{C}$ & -2.635 & 7.426 & -4.876 \\
\hline
\end{tabular}




\begin{tabular}{|c|c|c|c|c|c|c|c|}
\hline $\mathrm{C}$ & -3.618 & 7.558 & -3.962 & $\mathrm{C}$ & -3.666 & 7.506 & -3.927 \\
\hline $\mathrm{C}$ & -3.474 & 6.966 & -2.705 & $\mathrm{C}$ & -3.510 & 6.904 & -2.681 \\
\hline $\mathrm{C}$ & -3.791 & 8.688 & -6.558 & $\mathrm{C}$ & -3.843 & 8.693 & -6.508 \\
\hline $\mathrm{C}$ & -4.318 & 8.332 & 4.418 & $\mathrm{C}$ & -4.292 & 8.404 & 4.376 \\
\hline $\mathrm{H}$ & 0.657 & 0.348 & 1.340 & $\mathrm{H}$ & 0.513 & 0.310 & 1.415 \\
\hline $\mathrm{H}$ & 1.833 & -1.320 & 2.466 & $\mathrm{H}$ & 1.606 & -1.364 & 2.597 \\
\hline $\mathrm{H}$ & 2.637 & -3.624 & 2.734 & $\mathrm{H}$ & 2.420 & -3.655 & 2.861 \\
\hline $\mathrm{H}$ & 0.660 & -4.723 & -0.925 & $\mathrm{H}$ & 0.877 & -4.636 & -1.052 \\
\hline $\mathrm{H}$ & -0.122 & -2.428 & -1.202 & $\mathrm{H}$ & 0.062 & -2.361 & -1.310 \\
\hline $\mathrm{H}$ & 3.686 & -4.903 & -0.995 & $\mathrm{H}$ & 3.752 & -4.918 & -0.982 \\
\hline $\mathrm{H}$ & 4.294 & -6.231 & -2.970 & $\mathrm{H}$ & 4.343 & -6.287 & -2.936 \\
\hline $\mathrm{H}$ & 2.088 & -9.574 & -1.405 & $\mathrm{H}$ & 1.970 & -9.527 & -1.388 \\
\hline $\mathrm{H}$ & 1.447 & -8.212 & 0.575 & $\mathrm{H}$ & 1.363 & -8.135 & 0.583 \\
\hline $\mathrm{H}$ & 0.390 & -5.600 & 3.082 & $\mathrm{H}$ & 0.371 & -5.644 & 3.060 \\
\hline $\mathrm{H}$ & 0.659 & -6.619 & 5.333 & $\mathrm{H}$ & 0.655 & -6.705 & 5.291 \\
\hline $\mathrm{H}$ & 4.527 & -8.104 & 4.185 & $\mathrm{H}$ & 4.591 & -8.025 & 4.151 \\
\hline $\mathrm{H}$ & 4.271 & -7.064 & 1.974 & $\mathrm{H}$ & 4.313 & -6.962 & 1.952 \\
\hline $\mathrm{H}$ & 3.611 & -9.014 & 7.584 & $\mathrm{H}$ & 3.647 & -9.037 & 7.524 \\
\hline $\mathrm{H}$ & 4.697 & -8.068 & 6.529 & $\mathrm{H}$ & 4.731 & -8.050 & 6.505 \\
\hline $\mathrm{H}$ & 3.938 & -9.569 & 5.919 & $\mathrm{H}$ & 4.013 & -9.558 & 5.855 \\
\hline $\mathrm{H}$ & 4.415 & -9.148 & -5.137 & $\mathrm{H}$ & 4.354 & -9.238 & -5.075 \\
\hline $\mathrm{H}$ & 5.315 & -8.043 & -4.061 & $\mathrm{H}$ & 5.297 & -8.161 & -4.009 \\
\hline $\mathrm{H}$ & 3.849 & -7.470 & -4.910 & $\mathrm{H}$ & 3.865 & -7.531 & -4.885 \\
\hline $\mathrm{H}$ & -0.657 & -0.348 & -1.340 & $\mathrm{H}$ & -0.513 & -0.310 & -1.415 \\
\hline $\mathrm{H}$ & -1.833 & 1.320 & -2.466 & $\mathrm{H}$ & -1.606 & 1.364 & -2.597 \\
\hline $\mathrm{H}$ & -2.637 & 3.624 & -2.734 & $\mathrm{H}$ & -2.420 & 3.655 & -2.861 \\
\hline $\mathrm{H}$ & -0.660 & 4.723 & 0.925 & $\mathrm{H}$ & -0.877 & 4.636 & 1.052 \\
\hline $\mathrm{H}$ & 0.122 & 2.428 & 1.202 & $\mathrm{H}$ & -0.062 & 2.361 & 1.310 \\
\hline $\mathrm{H}$ & -3.686 & 4.903 & 0.995 & $\mathrm{H}$ & -3.752 & 4.918 & 0.982 \\
\hline $\mathrm{H}$ & -4.294 & 6.231 & 2.970 & $\mathrm{H}$ & -4.343 & 6.287 & 2.936 \\
\hline $\mathrm{H}$ & -2.088 & 9.574 & 1.405 & $\mathrm{H}$ & -1.970 & 9.527 & 1.388 \\
\hline $\mathrm{H}$ & -1.447 & 8.212 & -0.575 & $\mathrm{H}$ & -1.363 & 8.135 & -0.583 \\
\hline $\mathrm{H}$ & -0.390 & 5.600 & -3.082 & $\mathrm{H}$ & -0.371 & 5.644 & -3.060 \\
\hline $\mathrm{H}$ & -0.659 & 6.619 & -5.333 & $\mathrm{H}$ & -0.655 & 6.705 & -5.291 \\
\hline $\mathrm{H}$ & -4.527 & 8.104 & -4.185 & $\mathrm{H}$ & -4.591 & 8.025 & -4.151 \\
\hline $\mathrm{H}$ & -4.271 & 7.064 & -1.974 & $\mathrm{H}$ & -4.313 & 6.962 & -1.952 \\
\hline $\mathrm{H}$ & -3.611 & 9.014 & -7.584 & $\mathrm{H}$ & -3.647 & 9.037 & -7.524 \\
\hline $\mathrm{H}$ & -4.697 & 8.068 & -6.529 & $\mathrm{H}$ & -4.731 & 8.050 & -6.505 \\
\hline $\mathrm{H}$ & -3.938 & 9.569 & -5.919 & $\mathrm{H}$ & -4.013 & 9.558 & -5.855 \\
\hline $\mathrm{H}$ & -4.415 & 9.148 & 5.137 & $\mathrm{H}$ & -4.354 & 9.238 & 5.075 \\
\hline $\mathrm{H}$ & -5.315 & 8.043 & 4.061 & $\mathrm{H}$ & -5.297 & 8.161 & 4.009 \\
\hline $\mathrm{H}$ & -3.849 & 7.470 & 4.910 & $\mathrm{H}$ & -3.865 & 7.531 & 4.885 \\
\hline
\end{tabular}


Table S8. XYZ coordinates and energies (hr) for for the singlet, broken symmetry (BS), and triplet states of $\mathbf{1}^{2+}$.

\begin{tabular}{|c|c|c|c|c|c|c|c|c|c|c|c|}
\hline \multicolumn{4}{|c|}{$\begin{array}{l}\text { singlet } \\
(-2033.22342184 \mathrm{hr})\end{array}$} & \multicolumn{4}{|c|}{$\begin{array}{l}\text { BS } \\
(-2033.22949390 \mathrm{hr}) \\
\end{array}$} & \multicolumn{4}{|c|}{$\begin{array}{l}\text { triplet } \\
(-2033.22741720 \mathrm{hr})\end{array}$} \\
\hline $\mathrm{N}$ & 2.174 & -5.631 & 1.066 & $\mathrm{~N}$ & 2.226 & -5.624 & 1.058 & $\mathrm{~N}$ & 2.241 & -5.632 & 1.052 \\
\hline $\mathrm{O}$ & 3.449 & -8.850 & -3.318 & $\mathrm{O}$ & 3.558 & -8.775 & -3.317 & $\mathrm{O}$ & 3.592 & -8.755 & -3.335 \\
\hline $\mathrm{O}$ & 2.679 & -7.970 & 6.115 & $\mathrm{O}$ & 2.583 & -7.837 & 6.139 & $\mathrm{O}$ & 2.543 & -7.796 & 6.155 \\
\hline $\mathrm{C}$ & 0.317 & -0.294 & 0.531 & $\mathrm{C}$ & 0.391 & -0.267 & 0.489 & $\mathrm{C}$ & 0.421 & -0.257 & 0.464 \\
\hline $\mathrm{C}$ & 0.768 & -1.654 & 0.623 & $\mathrm{C}$ & 0.836 & -1.643 & 0.589 & $\mathrm{C}$ & 0.863 & -1.644 & 0.570 \\
\hline $\mathrm{C}$ & 1.437 & -2.083 & 1.799 & $\mathrm{C}$ & 1.665 & -2.012 & 1.675 & $\mathrm{C}$ & 1.754 & -1.992 & 1.609 \\
\hline $\mathrm{C}$ & 1.896 & -3.373 & 1.955 & $\mathrm{C}$ & 2.122 & -3.307 & 1.839 & $\mathrm{C}$ & 2.210 & -3.291 & 1.775 \\
\hline $\mathrm{C}$ & 1.717 & -4.329 & 0.924 & $\mathrm{C}$ & 1.770 & -4.301 & 0.902 & $\mathrm{C}$ & 1.786 & -4.299 & 0.890 \\
\hline $\mathrm{C}$ & 1.049 & -3.915 & -0.261 & $\mathrm{C}$ & 0.947 & -3.952 & -0.194 & $\mathrm{C}$ & 0.900 & -3.972 & -0.156 \\
\hline C & 0.590 & -2.625 & -0.399 & $\mathrm{C}$ & 0.491 & -2.657 & -0.339 & $\mathrm{C}$ & 0.449 & -2.672 & -0.307 \\
\hline $\mathrm{C}$ & 2.507 & -6.425 & -0.075 & $\mathrm{C}$ & 2.568 & -6.399 & -0.074 & $\mathrm{C}$ & 2.588 & -6.400 & -0.078 \\
\hline $\mathrm{C}$ & 3.348 & -5.922 & -1.075 & $\mathrm{C}$ & 3.280 & -5.825 & -1.144 & $\mathrm{C}$ & 3.267 & -5.809 & -1.163 \\
\hline $\mathrm{C}$ & 3.684 & -6.699 & -2.182 & $\mathrm{C}$ & 3.633 & -6.586 & -2.248 & $\mathrm{C}$ & 3.624 & -6.563 & -2.270 \\
\hline $\mathrm{C}$ & 3.186 & -8.006 & -2.293 & $\mathrm{C}$ & 3.271 & -7.948 & -2.306 & $\mathrm{C}$ & 3.299 & -7.935 & -2.320 \\
\hline $\mathrm{C}$ & 2.349 & -8.516 & -1.283 & $\mathrm{C}$ & 2.551 & -8.524 & -1.234 & $\mathrm{C}$ & 2.609 & -8.527 & -1.237 \\
\hline $\mathrm{C}$ & 2.014 & -7.737 & -0.189 & $\mathrm{C}$ & 2.210 & -7.765 & -0.134 & $\mathrm{C}$ & 2.263 & -7.777 & -0.135 \\
\hline C & 2.333 & -6.220 & 2.358 & $\mathrm{C}$ & 2.342 & -6.190 & 2.348 & $\mathrm{C}$ & 2.342 & -6.189 & 2.343 \\
\hline C & 1.297 & -6.152 & 3.309 & $\mathrm{C}$ & 1.359 & -5.939 & 3.334 & $\mathrm{C}$ & 1.373 & -5.893 & 3.331 \\
\hline $\mathrm{C}$ & 1.449 & -6.742 & 4.552 & $\mathrm{C}$ & 1.472 & -6.507 & 4.584 & $\mathrm{C}$ & 1.472 & -6.449 & 4.588 \\
\hline $\mathrm{C}$ & 2.635 & -7.426 & 4.876 & $\mathrm{C}$ & 2.571 & -7.341 & 4.898 & $\mathrm{C}$ & 2.544 & -7.315 & 4.908 \\
\hline C & 3.666 & -7.506 & 3.927 & $\mathrm{C}$ & 3.554 & -7.592 & 3.918 & $\mathrm{C}$ & 3.516 & -7.608 & 3.929 \\
\hline $\mathrm{C}$ & 3.510 & -6.904 & 2.681 & $\mathrm{C}$ & 3.434 & -7.024 & 2.660 & $\mathrm{C}$ & 3.411 & -7.052 & 2.665 \\
\hline $\mathrm{C}$ & 3.843 & -8.693 & 6.508 & $\mathrm{C}$ & 3.641 & -8.715 & 6.550 & $\mathrm{C}$ & 3.574 & -8.703 & 6.575 \\
\hline $\mathrm{C}$ & 4.292 & -8.404 & -4.376 & $\mathrm{C}$ & 4.282 & -8.289 & -4.456 & $\mathrm{C}$ & 4.285 & -8.252 & -4.486 \\
\hline $\mathrm{C}$ & -0.317 & 0.294 & -0.531 & $\mathrm{C}$ & -0.391 & 0.267 & -0.489 & $\mathrm{C}$ & -0.421 & 0.257 & -0.464 \\
\hline $\mathrm{N}$ & -2.174 & 5.631 & -1.066 & $\mathrm{~N}$ & -2.226 & 5.624 & -1.058 & $\mathrm{~N}$ & -2.241 & 5.632 & -1.052 \\
\hline $\mathrm{O}$ & -3.449 & 8.850 & 3.318 & $\mathrm{O}$ & -3.558 & 8.775 & 3.317 & $\mathrm{O}$ & -3.592 & 8.755 & 3.335 \\
\hline $\mathrm{O}$ & -2.679 & 7.970 & -6.115 & $\mathrm{O}$ & -2.582 & 7.837 & -6.139 & $\mathrm{O}$ & -2.543 & 7.796 & -6.155 \\
\hline $\mathrm{C}$ & -0.768 & 1.654 & -0.623 & $\mathrm{C}$ & -0.837 & 1.643 & -0.588 & $\mathrm{C}$ & -0.863 & 1.644 & -0.570 \\
\hline $\mathrm{C}$ & -1.437 & 2.083 & -1.799 & $\mathrm{C}$ & -1.666 & 2.012 & -1.675 & $\mathrm{C}$ & -1.754 & 1.992 & -1.609 \\
\hline $\mathrm{C}$ & -1.896 & 3.373 & -1.955 & $\mathrm{C}$ & -2.122 & 3.307 & -1.839 & $\mathrm{C}$ & -2.210 & 3.291 & -1.775 \\
\hline $\mathrm{C}$ & -1.717 & 4.329 & -0.924 & $\mathrm{C}$ & -1.770 & 4.301 & -0.902 & $\mathrm{C}$ & -1.786 & 4.299 & -0.890 \\
\hline C & -1.049 & 3.915 & 0.261 & $\mathrm{C}$ & -0.948 & 3.952 & 0.194 & $\mathrm{C}$ & -0.900 & 3.972 & 0.156 \\
\hline $\mathrm{C}$ & -0.590 & 2.625 & 0.399 & $\mathrm{C}$ & -0.491 & 2.657 & 0.339 & $\mathrm{C}$ & -0.449 & 2.672 & 0.307 \\
\hline $\mathrm{C}$ & -2.507 & 6.425 & 0.075 & $\mathrm{C}$ & -2.568 & 6.399 & 0.074 & $\mathrm{C}$ & -2.588 & 6.400 & 0.078 \\
\hline $\mathrm{C}$ & -3.348 & 5.922 & 1.075 & $\mathrm{C}$ & -3.281 & 5.825 & 1.144 & $\mathrm{C}$ & -3.267 & 5.809 & 1.163 \\
\hline C & -3.684 & 6.699 & 2.182 & $\mathrm{C}$ & -3.633 & 6.586 & 2.248 & $\mathrm{C}$ & -3.624 & 6.563 & 2.270 \\
\hline $\mathrm{C}$ & -3.186 & 8.006 & 2.293 & $\mathrm{C}$ & -3.271 & 7.948 & 2.306 & $\mathrm{C}$ & -3.299 & 7.935 & 2.320 \\
\hline $\mathrm{C}$ & -2.349 & 8.516 & 1.283 & $\mathrm{C}$ & -2.551 & 8.524 & 1.234 & $\mathrm{C}$ & -2.609 & 8.527 & 1.237 \\
\hline $\mathrm{C}$ & -2.014 & 7.737 & 0.189 & $\mathrm{C}$ & -2.210 & 7.765 & 0.134 & $\mathrm{C}$ & -2.263 & 7.777 & 0.135 \\
\hline $\mathrm{C}$ & -2.333 & 6.220 & -2.358 & $\mathrm{C}$ & -2.342 & 6.190 & -2.348 & $\mathrm{C}$ & -2.342 & 6.189 & -2.343 \\
\hline $\mathrm{C}$ & -1.297 & 6.152 & -3.309 & $\mathrm{C}$ & -1.359 & 5.939 & -3.334 & $\mathrm{C}$ & -1.373 & 5.893 & -3.331 \\
\hline C & -1.449 & 6.742 & -4.552 & $\mathrm{C}$ & -1.472 & 6.507 & -4.584 & $\mathrm{C}$ & -1.472 & 6.449 & -4.588 \\
\hline $\mathrm{C}$ & -2.635 & 7.426 & -4.876 & $\mathrm{C}$ & -2.571 & 7.341 & -4.898 & $\mathrm{C}$ & -2.544 & 7.315 & -4.908 \\
\hline C & -3.666 & 7.506 & -3.927 & $\mathrm{C}$ & -3.554 & 7.592 & -3.918 & $\mathrm{C}$ & -3.516 & 7.608 & -3.929 \\
\hline $\mathrm{C}$ & -3.510 & 6.904 & -2.681 & $\mathrm{C}$ & -3.434 & 7.024 & -2.660 & $\mathrm{C}$ & -3.411 & 7.052 & -2.665 \\
\hline
\end{tabular}




\begin{tabular}{|c|c|c|c|c|c|c|c|c|c|c|c|}
\hline $\mathrm{C}$ & -3.843 & 8.693 & -6.508 & $\mathrm{C}$ & -3.640 & 8.715 & -6.550 & $\mathrm{C}$ & -3.574 & 8.703 & -6.575 \\
\hline $\mathrm{C}$ & -4.292 & 8.404 & 4.376 & $\mathrm{C}$ & -4.282 & 8.289 & 4.455 & $\mathrm{C}$ & -4.285 & 8.252 & 4.486 \\
\hline $\mathrm{H}$ & 0.513 & 0.310 & 1.415 & $\mathrm{H}$ & 0.734 & 0.377 & 1.296 & $\mathrm{H}$ & 0.832 & 0.403 & 1.225 \\
\hline $\mathrm{H}$ & 1.606 & -1.364 & 2.597 & $\mathrm{H}$ & 1.964 & -1.254 & 2.394 & $\mathrm{H}$ & 2.102 & -1.221 & 2.290 \\
\hline $\mathrm{H}$ & 2.420 & -3.655 & 2.861 & $\mathrm{H}$ & 2.775 & -3.554 & 2.668 & $\mathrm{H}$ & 2.910 & -3.526 & 2.569 \\
\hline $\mathrm{H}$ & 0.877 & -4.636 & -1.052 & $\mathrm{H}$ & 0.647 & -4.716 & -0.902 & $\mathrm{H}$ & 0.550 & -4.750 & -0.826 \\
\hline $\mathrm{H}$ & 0.062 & -2.361 & -1.310 & $\mathrm{H}$ & -0.161 & -2.431 & -1.176 & $\mathrm{H}$ & -0.249 & -2.458 & -1.109 \\
\hline $\mathrm{H}$ & 3.752 & -4.918 & -0.982 & $\mathrm{H}$ & 3.587 & -4.785 & -1.091 & $\mathrm{H}$ & 3.545 & -4.761 & -1.117 \\
\hline $\mathrm{H}$ & 4.343 & -6.287 & -2.936 & $\mathrm{H}$ & 4.202 & -6.129 & -3.049 & $\mathrm{H}$ & 4.167 & -6.092 & -3.080 \\
\hline $\mathrm{H}$ & 1.970 & -9.527 & -1.388 & $\mathrm{H}$ & 2.266 & -9.567 & -1.307 & $\mathrm{H}$ & 2.347 & -9.578 & -1.307 \\
\hline $\mathrm{H}$ & 1.363 & -8.135 & 0.583 & $\mathrm{H}$ & 1.638 & -8.209 & 0.674 & $\mathrm{H}$ & 1.712 & -8.234 & 0.680 \\
\hline $\mathrm{H}$ & 0.371 & -5.644 & 3.060 & $\mathrm{H}$ & 0.498 & -5.326 & 3.094 & $\mathrm{H}$ & 0.533 & -5.253 & 3.088 \\
\hline $\mathrm{H}$ & 0.655 & -6.705 & 5.291 & $\mathrm{H}$ & 0.716 & -6.346 & 5.345 & $\mathrm{H}$ & 0.725 & -6.253 & 5.349 \\
\hline $\mathrm{H}$ & 4.591 & -8.025 & 4.151 & $\mathrm{H}$ & 4.414 & -8.212 & 4.141 & $\mathrm{H}$ & 4.359 & -8.250 & 4.158 \\
\hline $\mathrm{H}$ & 4.313 & -6.962 & 1.952 & $\mathrm{H}$ & 4.205 & -7.199 & 1.917 & $\mathrm{H}$ & 4.177 & -7.257 & 1.925 \\
\hline $\mathrm{H}$ & 3.647 & -9.037 & 7.524 & $\mathrm{H}$ & 3.409 & -8.984 & 7.580 & $\mathrm{H}$ & 3.335 & -8.952 & 7.608 \\
\hline $\mathrm{H}$ & 4.731 & -8.050 & 6.505 & $\mathrm{H}$ & 4.608 & -8.203 & 6.510 & $\mathrm{H}$ & 4.556 & -8.222 & 6.528 \\
\hline $\mathrm{H}$ & 4.013 & -9.558 & 5.855 & $\mathrm{H}$ & 3.662 & -9.616 & 5.927 & $\mathrm{H}$ & 3.565 & -9.610 & 5.963 \\
\hline $\mathrm{H}$ & 4.354 & -9.238 & -5.075 & $\mathrm{H}$ & 4.376 & -9.144 & -5.124 & $\mathrm{H}$ & 4.392 & -9.106 & -5.153 \\
\hline $\mathrm{H}$ & 5.297 & -8.161 & -4.009 & $\mathrm{H}$ & 5.277 & -7.938 & -4.163 & $\mathrm{H}$ & 5.275 & -7.873 & -4.209 \\
\hline $\mathrm{H}$ & 3.865 & -7.531 & -4.885 & $\mathrm{H}$ & 3.729 & -7.488 & -4.956 & $\mathrm{H}$ & 3.702 & -7.467 & -4.979 \\
\hline $\mathrm{H}$ & -0.513 & -0.310 & -1.415 & $\mathrm{H}$ & -0.735 & -0.377 & -1.296 & $\mathrm{H}$ & -0.832 & -0.403 & -1.225 \\
\hline $\mathrm{H}$ & -1.606 & 1.364 & -2.597 & $\mathrm{H}$ & -1.964 & 1.254 & -2.394 & $\mathrm{H}$ & -2.102 & 1.221 & -2.290 \\
\hline $\mathrm{H}$ & -2.420 & 3.655 & -2.861 & $\mathrm{H}$ & -2.775 & 3.554 & -2.668 & $\mathrm{H}$ & -2.910 & 3.526 & -2.569 \\
\hline $\mathrm{H}$ & -0.877 & 4.636 & 1.052 & $\mathrm{H}$ & -0.647 & 4.716 & 0.903 & $\mathrm{H}$ & -0.550 & 4.750 & 0.826 \\
\hline $\mathrm{H}$ & -0.062 & 2.361 & 1.310 & $\mathrm{H}$ & 0.161 & 2.431 & 1.176 & $\mathrm{H}$ & 0.249 & 2.458 & 1.109 \\
\hline $\mathrm{H}$ & -3.752 & 4.918 & 0.982 & $\mathrm{H}$ & -3.588 & 4.785 & 1.091 & $\mathrm{H}$ & -3.545 & 4.761 & 1.117 \\
\hline $\mathrm{H}$ & -4.343 & 6.287 & 2.936 & $\mathrm{H}$ & -4.203 & 6.129 & 3.048 & $\mathrm{H}$ & -4.167 & 6.092 & 3.080 \\
\hline $\mathrm{H}$ & -1.970 & 9.527 & 1.388 & $\mathrm{H}$ & -2.266 & 9.567 & 1.307 & $\mathrm{H}$ & -2.347 & 9.578 & 1.307 \\
\hline $\mathrm{H}$ & -1.363 & 8.135 & -0.583 & $\mathrm{H}$ & -1.638 & 8.209 & -0.674 & $\mathrm{H}$ & -1.712 & 8.234 & -0.680 \\
\hline $\mathrm{H}$ & -0.371 & 5.644 & -3.060 & $\mathrm{H}$ & -0.498 & 5.326 & -3.094 & $\mathrm{H}$ & -0.533 & 5.253 & -3.088 \\
\hline $\mathrm{H}$ & -0.655 & 6.705 & -5.291 & $\mathrm{H}$ & -0.716 & 6.346 & -5.345 & $\mathrm{H}$ & -0.725 & 6.253 & -5.349 \\
\hline $\mathrm{H}$ & -4.591 & 8.025 & -4.151 & $\mathrm{H}$ & -4.414 & 8.212 & -4.141 & $\mathrm{H}$ & -4.359 & 8.250 & -4.158 \\
\hline $\mathrm{H}$ & -4.313 & 6.962 & -1.952 & $\mathrm{H}$ & -4.205 & 7.199 & -1.917 & $\mathrm{H}$ & -4.177 & 7.257 & -1.925 \\
\hline $\mathrm{H}$ & -3.647 & 9.037 & -7.524 & $\mathrm{H}$ & -3.408 & 8.984 & -7.580 & $\mathrm{H}$ & -3.335 & 8.952 & -7.608 \\
\hline $\mathrm{H}$ & -4.731 & 8.050 & -6.505 & $\mathrm{H}$ & -4.607 & 8.203 & -6.510 & $\mathrm{H}$ & -4.556 & 8.222 & -6.528 \\
\hline $\mathrm{H}$ & -4.013 & 9.558 & -5.855 & $\mathrm{H}$ & -3.661 & 9.616 & -5.927 & $\mathrm{H}$ & -3.565 & 9.610 & -5.963 \\
\hline $\mathrm{H}$ & -4.354 & 9.238 & 5.075 & $\mathrm{H}$ & -4.377 & 9.144 & 5.124 & $\mathrm{H}$ & -4.392 & 9.106 & 5.153 \\
\hline $\mathrm{H}$ & -5.297 & 8.161 & 4.009 & $\mathrm{H}$ & -5.277 & 7.938 & 4.162 & $\mathrm{H}$ & -5.275 & 7.873 & 4.209 \\
\hline $\mathrm{H}$ & -3.865 & 7.531 & 4.885 & $\mathrm{H}$ & -3.729 & 7.488 & 4.956 & $\mathrm{H}$ & -3.702 & 7.467 & 4.979 \\
\hline
\end{tabular}


Table S9. XYZ coordinates and energies (hr) for $\mathbf{2}$ and $\mathbf{2}^{+}$.

\begin{tabular}{|c|c|c|c|c|c|c|c|}
\hline \multicolumn{4}{|c|}{$\begin{array}{l}\mathbf{2} \\
(-3127.84493575 \mathrm{hr})\end{array}$} & \multicolumn{4}{|c|}{$\begin{array}{l}\mathbf{2}^{+} \\
(-3127.66457753 \mathrm{hr})\end{array}$} \\
\hline$S$ & -0.105 & 0.346 & -0.090 & $\mathrm{~S}$ & 0.095 & -0.304 & -0.058 \\
\hline $\mathrm{O}$ & 10.244 & -6.088 & 2.851 & $\mathrm{O}$ & -10.261 & 6.326 & 2.249 \\
\hline $\mathrm{O}$ & 12.473 & 1.587 & -2.638 & $\mathrm{O}$ & -12.533 & -1.880 & -2.138 \\
\hline $\mathrm{O}$ & 1.388 & 3.962 & -0.220 & $\mathrm{O}$ & -1.400 & -3.925 & -0.214 \\
\hline $\mathrm{O}$ & -1.566 & 3.971 & -0.226 & $\mathrm{O}$ & 1.585 & -3.917 & -0.241 \\
\hline $\mathrm{O}$ & -12.750 & 1.796 & 2.412 & $\mathrm{O}$ & 12.861 & -2.100 & 1.526 \\
\hline $\mathrm{O}$ & -10.584 & -6.023 & -2.764 & $\mathrm{O}$ & 10.480 & 6.395 & -1.991 \\
\hline $\mathrm{N}$ & 8.647 & -1.484 & 0.077 & $\mathrm{~N}$ & -8.610 & 1.457 & 0.043 \\
\hline $\mathrm{N}$ & -8.889 & -1.363 & -0.146 & $\mathrm{~N}$ & 8.817 & 1.393 & -0.121 \\
\hline $\mathrm{C}$ & 1.174 & 1.555 & -0.119 & $\mathrm{C}$ & -1.175 & -1.523 & -0.109 \\
\hline $\mathrm{C}$ & 0.622 & 2.825 & -0.170 & $\mathrm{C}$ & -0.616 & -2.813 & -0.166 \\
\hline $\mathrm{C}$ & -0.804 & 2.832 & -0.195 & $\mathrm{C}$ & 0.792 & -2.817 & -0.192 \\
\hline $\mathrm{C}$ & -1.370 & 1.569 & -0.154 & $\mathrm{C}$ & 1.358 & -1.528 & -0.129 \\
\hline $\mathrm{C}$ & 2.577 & 1.242 & -0.095 & $\mathrm{C}$ & -2.560 & -1.238 & -0.089 \\
\hline $\mathrm{C}$ & 3.125 & 0.005 & -0.022 & $\mathrm{C}$ & -3.124 & 0.013 & -0.017 \\
\hline $\mathrm{C}$ & 4.543 & -0.331 & 0.004 & $\mathrm{C}$ & -4.520 & 0.334 & 0.002 \\
\hline $\mathrm{C}$ & 4.931 & -1.685 & 0.036 & $\mathrm{C}$ & -4.919 & 1.694 & 0.047 \\
\hline $\mathrm{C}$ & 6.264 & -2.072 & 0.052 & $\mathrm{C}$ & -6.245 & 2.071 & 0.063 \\
\hline $\mathrm{C}$ & 7.288 & -1.107 & 0.052 & $\mathrm{C}$ & -7.272 & 1.094 & 0.031 \\
\hline $\mathrm{C}$ & 6.915 & 0.253 & 0.031 & $\mathrm{C}$ & -6.887 & -0.273 & -0.011 \\
\hline $\mathrm{C}$ & 5.580 & 0.625 & 0.002 & $\mathrm{C}$ & -5.559 & -0.635 & -0.022 \\
\hline $\mathrm{C}$ & 9.058 & -2.668 & 0.756 & $\mathrm{C}$ & -9.030 & 2.717 & 0.579 \\
\hline $\mathrm{C}$ & 8.615 & -2.940 & 2.063 & $\mathrm{C}$ & -8.631 & 3.115 & 1.867 \\
\hline $\mathrm{C}$ & 9.020 & -4.090 & 2.725 & $\mathrm{C}$ & -9.054 & 4.327 & 2.389 \\
\hline $\mathrm{C}$ & 9.899 & -4.993 & 2.108 & $\mathrm{C}$ & -9.900 & 5.166 & 1.643 \\
\hline C & 10.357 & -4.727 & 0.813 & $\mathrm{C}$ & -10.311 & 4.768 & 0.363 \\
\hline $\mathrm{C}$ & 9.927 & -3.578 & 0.145 & $\mathrm{C}$ & -9.872 & 3.553 & -0.160 \\
\hline $\mathrm{C}$ & 11.125 & -7.036 & 2.272 & $\mathrm{C}$ & -11.124 & 7.219 & 1.553 \\
\hline $\mathrm{C}$ & 9.630 & -0.696 & -0.588 & $\mathrm{C}$ & -9.618 & 0.590 & -0.487 \\
\hline $\mathrm{C}$ & 10.833 & -0.368 & 0.047 & $\mathrm{C}$ & -10.762 & 0.299 & 0.262 \\
\hline $\mathrm{C}$ & 11.814 & 0.383 & -0.606 & $\mathrm{C}$ & -11.765 & -0.521 & -0.255 \\
\hline $\mathrm{C}$ & 11.590 & 0.839 & -1.909 & $\mathrm{C}$ & -11.625 & -1.069 & -1.539 \\
\hline $\mathrm{C}$ & 10.383 & 0.522 & -2.550 & $\mathrm{C}$ & -10.477 & -0.776 & -2.294 \\
\hline $\mathrm{C}$ & 9.423 & -0.243 & -1.904 & $\mathrm{C}$ & -9.490 & 0.049 & -1.778 \\
\hline $\mathrm{C}$ & 13.705 & 1.941 & -2.034 & $\mathrm{C}$ & -13.726 & -2.207 & -1.435 \\
\hline $\mathrm{C}$ & 1.398 & 4.731 & 1.003 & $\mathrm{C}$ & -1.373 & -4.750 & 0.983 \\
\hline $\mathrm{C}$ & 2.319 & 5.929 & 0.809 & $\mathrm{C}$ & -2.289 & -5.945 & 0.760 \\
\hline $\mathrm{C}$ & 3.785 & 5.584 & 0.504 & $\mathrm{C}$ & -3.765 & -5.603 & 0.499 \\
\hline $\mathrm{C}$ & 4.517 & 4.862 & 1.642 & $\mathrm{C}$ & -4.474 & -4.915 & 1.671 \\
\hline $\mathrm{C}$ & -1.521 & 4.703 & -1.472 & $\mathrm{C}$ & 1.412 & -4.794 & -1.390 \\
\hline $\mathrm{C}$ & -2.632 & 5.746 & -1.451 & $\mathrm{C}$ & 2.588 & -5.759 & -1.422 \\
\hline $\mathrm{C}$ & -2.510 & 6.810 & -0.348 & $\mathrm{C}$ & 2.693 & -6.706 & -0.215 \\
\hline $\mathrm{C}$ & -1.339 & 7.782 & -0.538 & $\mathrm{C}$ & 1.579 & -7.756 & -0.137 \\
\hline $\mathrm{C}$ & -2.777 & 1.274 & -0.159 & $\mathrm{C}$ & 2.744 & -1.252 & -0.135 \\
\hline $\mathrm{C}$ & -3.344 & 0.042 & -0.145 & $\mathrm{C}$ & 3.319 & -0.004 & -0.087 \\
\hline $\mathrm{C}$ & -4.766 & -0.273 & -0.148 & $\mathrm{C}$ & 4.717 & 0.304 & -0.096 \\
\hline $\mathrm{C}$ & -5.175 & -1.621 & -0.125 & $\mathrm{C}$ & 5.129 & 1.660 & -0.031 \\
\hline
\end{tabular}




\begin{tabular}{|c|c|c|c|c|c|c|c|}
\hline $\mathrm{C}$ & -6.515 & -1.987 & -0.116 & $\mathrm{C}$ & 6.458 & 2.026 & -0.039 \\
\hline $\mathrm{C}$ & -7.523 & -1.007 & -0.145 & C & 7.476 & 1.041 & -0.110 \\
\hline $\mathrm{C}$ & -7.129 & 0.346 & -0.177 & $\mathrm{C}$ & 7.078 & -0.322 & -0.173 \\
\hline $\mathrm{C}$ & -5.789 & 0.699 & -0.172 & $\mathrm{C}$ & 5.746 & -0.672 & -0.171 \\
\hline $\mathrm{C}$ & -9.849 & -0.554 & 0.528 & $\mathrm{C}$ & 9.827 & 0.480 & 0.321 \\
\hline C & -11.067 & -0.225 & -0.092 & $\mathrm{C}$ & 10.952 & 0.231 & -0.482 \\
\hline $\mathrm{C}$ & -12.012 & 0.548 & 0.566 & $\mathrm{C}$ & 11.945 & -0.633 & -0.048 \\
\hline $\mathrm{C}$ & -11.760 & 1.034 & 1.858 & $\mathrm{C}$ & 11.836 & -1.274 & 1.199 \\
\hline $\mathrm{C}$ & -10.547 & 0.721 & 2.482 & C & 10.717 & -1.027 & 2.005 \\
\hline $\mathrm{C}$ & -9.610 & -0.077 & 1.822 & $\mathrm{C}$ & 9.726 & -0.149 & 1.566 \\
\hline $\mathrm{C}$ & -12.546 & 2.311 & 3.717 & $\mathrm{C}$ & 12.822 & -2.774 & 2.779 \\
\hline C & -9.325 & -2.561 & -0.783 & $\mathrm{C}$ & 9.239 & 2.686 & -0.569 \\
\hline $\mathrm{C}$ & -10.208 & -3.435 & -0.141 & $\mathrm{C}$ & 10.121 & 3.447 & 0.204 \\
\hline $\mathrm{C}$ & -10.662 & -4.596 & -0.770 & $\mathrm{C}$ & 10.564 & 4.693 & -0.237 \\
\hline C & -10.214 & -4.912 & -2.057 & $\mathrm{C}$ & 10.118 & 5.197 & -1.467 \\
\hline $\mathrm{C}$ & -9.321 & -4.045 & -2.705 & $\mathrm{C}$ & 9.233 & 4.433 & -2.247 \\
\hline C & -8.892 & -2.883 & -2.082 & $\mathrm{C}$ & 8.805 & 3.190 & -1.808 \\
\hline $\mathrm{C}$ & -11.483 & -6.933 & -2.154 & $\mathrm{C}$ & 11.386 & 7.214 & -1.261 \\
\hline $\mathrm{H}$ & 3.216 & 2.120 & -0.153 & $\mathrm{H}$ & -3.193 & -2.119 & -0.148 \\
\hline $\mathrm{H}$ & 2.455 & -0.854 & 0.022 & $\mathrm{H}$ & -2.454 & 0.872 & 0.028 \\
\hline $\mathrm{H}$ & 4.161 & -2.454 & 0.033 & $\mathrm{H}$ & -4.153 & 2.465 & 0.054 \\
\hline $\mathrm{H}$ & 6.521 & -3.126 & 0.061 & $\mathrm{H}$ & -6.508 & 3.122 & 0.080 \\
\hline $\mathrm{H}$ & 7.686 & 1.016 & 0.045 & $\mathrm{H}$ & -7.653 & -1.040 & -0.013 \\
\hline $\mathrm{H}$ & 5.339 & 1.684 & -0.004 & $\mathrm{H}$ & -5.311 & -1.692 & -0.032 \\
\hline $\mathrm{H}$ & 7.945 & -2.241 & 2.554 & $\mathrm{H}$ & -7.993 & 2.464 & 2.457 \\
\hline $\mathrm{H}$ & 8.678 & -4.304 & 3.733 & $\mathrm{H}$ & -8.758 & 4.643 & 3.384 \\
\hline $\mathrm{H}$ & 11.034 & -5.407 & 0.309 & $\mathrm{H}$ & -10.961 & 5.396 & -0.235 \\
\hline $\mathrm{H}$ & 10.278 & -3.384 & -0.864 & $\mathrm{H}$ & -10.188 & 3.250 & -1.154 \\
\hline $\mathrm{H}$ & 11.264 & -7.815 & 3.024 & $\mathrm{H}$ & -11.278 & 8.064 & 2.225 \\
\hline $\mathrm{H}$ & 12.098 & -6.590 & 2.029 & $\mathrm{H}$ & -12.090 & 6.749 & 1.331 \\
\hline $\mathrm{H}$ & 10.698 & -7.483 & 1.364 & $\mathrm{H}$ & -10.666 & 7.572 & 0.621 \\
\hline $\mathrm{H}$ & 11.008 & -0.709 & 1.062 & $\mathrm{H}$ & -10.871 & 0.717 & 1.258 \\
\hline $\mathrm{H}$ & 12.734 & 0.614 & -0.082 & $\mathrm{H}$ & -12.639 & -0.731 & 0.349 \\
\hline $\mathrm{H}$ & 10.226 & 0.874 & -3.565 & $\mathrm{H}$ & -10.392 & -1.196 & -3.291 \\
\hline $\mathrm{H}$ & 8.498 & -0.494 & -2.414 & $\mathrm{H}$ & -8.614 & 0.285 & -2.375 \\
\hline $\mathrm{H}$ & 14.247 & 2.527 & -2.779 & $\mathrm{H}$ & -14.297 & -2.853 & -2.104 \\
\hline $\mathrm{H}$ & 14.297 & 1.054 & -1.772 & $\mathrm{H}$ & -14.314 & -1.309 & -1.207 \\
\hline $\mathrm{H}$ & 13.555 & 2.551 & -1.133 & $\mathrm{H}$ & -13.508 & -2.748 & -0.506 \\
\hline $\mathrm{H}$ & 1.740 & 4.087 & 1.823 & $\mathrm{H}$ & -1.701 & -4.138 & 1.832 \\
\hline $\mathrm{H}$ & 0.377 & 5.059 & 1.233 & $\mathrm{H}$ & -0.344 & -5.075 & 1.172 \\
\hline $\mathrm{H}$ & 1.915 & 6.546 & -0.004 & $\mathrm{H}$ & -1.897 & -6.531 & -0.081 \\
\hline $\mathrm{H}$ & 2.262 & 6.538 & 1.723 & $\mathrm{H}$ & -2.207 & -6.584 & 1.651 \\
\hline $\mathrm{H}$ & 4.316 & 6.517 & 0.273 & $\mathrm{H}$ & -4.291 & -6.536 & 0.260 \\
\hline $\mathrm{H}$ & 3.824 & 4.975 & -0.408 & $\mathrm{H}$ & -3.833 & -4.977 & -0.400 \\
\hline $\mathrm{H}$ & 5.570 & 4.698 & 1.387 & $\mathrm{H}$ & -5.535 & -4.758 & 1.446 \\
\hline $\mathrm{H}$ & 4.077 & 3.881 & 1.856 & $\mathrm{H}$ & -4.040 & -3.935 & 1.901 \\
\hline $\mathrm{H}$ & 4.488 & 5.448 & 2.569 & $\mathrm{H}$ & -4.418 & -5.523 & 2.583 \\
\hline $\mathrm{H}$ & -1.676 & 4.000 & -2.301 & $\mathrm{H}$ & 1.392 & -4.176 & -2.296 \\
\hline $\mathrm{H}$ & -0.529 & 5.154 & -1.591 & $\mathrm{H}$ & 0.451 & -5.311 & -1.308 \\
\hline $\mathrm{H}$ & -3.588 & 5.219 & -1.344 & $\mathrm{H}$ & 3.510 & -5.169 & -1.510 \\
\hline
\end{tabular}




\begin{tabular}{lccc:cccc}
$\mathrm{H}$ & -2.649 & 6.233 & -2.437 & $\mathrm{H}$ & 2.503 & -6.345 & -2.348 \\
$\mathrm{H}$ & -3.446 & 7.383 & -0.318 & $\mathrm{H}$ & 3.661 & -7.220 & -0.269 \\
$\mathrm{H}$ & -2.425 & 6.307 & 0.622 & $\mathrm{H}$ & 2.714 & -6.112 & 0.706 \\
$\mathrm{H}$ & -1.317 & 8.531 & 0.262 & $\mathrm{H}$ & 1.727 & -8.420 & 0.721 \\
$\mathrm{H}$ & -1.419 & 8.317 & -1.492 & $\mathrm{H}$ & 1.557 & -8.379 & -1.039 \\
$\mathrm{H}$ & -0.373 & 7.265 & -0.529 & $\mathrm{H}$ & 0.588 & -7.300 & -0.028 \\
$\mathrm{H}$ & -3.402 & 2.164 & -0.170 & $\mathrm{H}$ & 3.368 & -2.140 & -0.179 \\
$\mathrm{H}$ & -2.686 & -0.827 & -0.131 & $\mathrm{H}$ & 2.656 & 0.859 & -0.037 \\
$\mathrm{H}$ & -4.417 & -2.401 & -0.098 & $\mathrm{H}$ & 4.370 & 2.436 & 0.043 \\
$\mathrm{H}$ & -6.788 & -3.036 & -0.084 & $\mathrm{H}$ & 6.732 & 3.072 & 0.028 \\
$\mathrm{H}$ & -7.889 & 1.120 & -0.210 & $\mathrm{H}$ & 7.836 & -1.093 & -0.248 \\
$\mathrm{H}$ & -5.532 & 1.754 & -0.202 & $\mathrm{H}$ & 5.488 & -1.724 & -0.243 \\
$\mathrm{H}$ & -11.267 & -0.586 & -1.096 & $\mathrm{H}$ & 11.040 & 0.718 & -1.448 \\
$\mathrm{H}$ & -12.954 & 0.804 & 0.091 & $\mathrm{H}$ & 12.817 & -0.837 & -0.661 \\
$\mathrm{H}$ & -10.324 & 1.076 & 3.482 & $\mathrm{H}$ & 10.613 & -1.497 & 2.976 \\
$\mathrm{H}$ & -8.677 & -0.327 & 2.318 & $\mathrm{H}$ & 8.869 & 0.054 & 2.202 \\
$\mathrm{H}$ & -13.444 & 2.883 & 3.958 & $\mathrm{H}$ & 13.733 & -3.372 & 2.822 \\
$\mathrm{H}$ & -11.672 & 2.975 & 3.758 & $\mathrm{H}$ & 11.949 & -3.434 & 2.851 \\
$\mathrm{H}$ & -12.420 & 1.507 & 4.455 & $\mathrm{H}$ & 12.813 & -2.063 & 3.614 \\
$\mathrm{H}$ & -10.551 & -3.202 & 0.863 & $\mathrm{H}$ & 10.464 & 3.062 & 1.159 \\
$\mathrm{H}$ & -11.350 & -5.246 & -0.242 & $\mathrm{H}$ & 11.246 & 5.260 & 0.385 \\
$\mathrm{H}$ & -8.988 & -4.297 & -3.708 & $\mathrm{H}$ & 8.910 & 4.831 & -3.203 \\
$\mathrm{H}$ & -8.212 & -2.212 & -2.599 & $\mathrm{H}$ & 8.137 & 2.598 & -2.426 \\
$\mathrm{H}$ & -11.642 & -7.731 & -2.881 & $\mathrm{H}$ & 11.534 & 8.107 & -1.869 \\
$\mathrm{H}$ & -12.446 & -6.457 & -1.923 & $\mathrm{H}$ & 12.349 & 6.711 & -1.112 \\
$\mathrm{H}$ & -11.065 & -7.360 & -1.232 & $\mathrm{H}$ & 10.969 & 7.502 & -0.287 \\
\hline \hline
\end{tabular}


Table S10. XYZ coordinates and energies (hr) for for the singlet, broken symmetry (BS), and triplet states of $2^{2+}$.

\begin{tabular}{|c|c|c|c|c|c|c|c|c|c|c|c|}
\hline \multicolumn{4}{|c|}{$\begin{array}{l}\text { singlet } \\
(-3127.40170528 \mathrm{hr})\end{array}$} & \multicolumn{4}{|c|}{$\begin{array}{l}\text { BS } \\
(-3127.40424931 \mathrm{hr}) \\
\end{array}$} & \multicolumn{4}{|c|}{$\begin{array}{l}\text { triplet } \\
(-3127.40073782 \mathrm{hr})\end{array}$} \\
\hline $\mathrm{S}$ & 0.084 & -0.298 & 0.067 & $\mathrm{~S}$ & 0.102 & -0.197 & -0.032 & $\mathrm{~S}$ & -0.099 & 0.184 & -0.030 \\
\hline $\mathrm{O}$ & -10.343 & 6.128 & 2.406 & $\mathrm{O}$ & -10.409 & 5.969 & 2.687 & $\mathrm{O}$ & 10.436 & -5.773 & 3.053 \\
\hline $\mathrm{O}$ & -12.397 & -1.824 & -2.458 & $\mathrm{O}$ & -12.352 & -1.836 & -2.560 & $\mathrm{O}$ & 12.271 & 1.587 & -2.922 \\
\hline $\mathrm{O}$ & -1.392 & -3.942 & -0.158 & $\mathrm{O}$ & -1.380 & -3.822 & -0.195 & $\mathrm{O}$ & 1.404 & 3.792 & -0.227 \\
\hline $\mathrm{O}$ & 1.634 & -3.860 & -0.270 & $\mathrm{O}$ & 1.619 & -3.794 & -0.244 & $\mathrm{O}$ & -1.589 & 3.789 & -0.253 \\
\hline $\mathrm{O}$ & 12.866 & -2.117 & 1.154 & $\mathrm{O}$ & 12.780 & -1.973 & 1.852 & $\mathrm{O}$ & -12.683 & 1.881 & 2.330 \\
\hline $\mathrm{O}$ & 10.354 & 6.480 & -1.850 & $\mathrm{O}$ & 10.523 & 6.243 & -2.364 & $\mathrm{O}$ & -10.615 & -5.922 & -2.793 \\
\hline $\mathrm{N}$ & -8.586 & 1.403 & 0.023 & $\mathrm{~N}$ & -8.644 & 1.392 & 0.054 & $\mathrm{~N}$ & 8.674 & -1.430 & 0.059 \\
\hline $\mathrm{N}$ & 8.757 & 1.415 & -0.156 & $\mathrm{~N}$ & 8.843 & 1.433 & -0.121 & $\mathrm{~N}$ & -8.885 & -1.370 & -0.108 \\
\hline $\mathrm{C}$ & -1.174 & -1.533 & -0.015 & $\mathrm{C}$ & -1.157 & -1.425 & -0.088 & $\mathrm{C}$ & 1.166 & 1.401 & -0.093 \\
\hline $\mathrm{C}$ & -0.607 & -2.835 & -0.104 & $\mathrm{C}$ & -0.597 & -2.716 & -0.150 & $\mathrm{C}$ & 0.621 & 2.685 & -0.164 \\
\hline $\mathrm{C}$ & 0.790 & -2.832 & -0.159 & $\mathrm{C}$ & 0.814 & -2.713 & -0.183 & $\mathrm{C}$ & -0.802 & 2.690 & -0.192 \\
\hline $\mathrm{C}$ & 1.352 & -1.514 & -0.066 & $\mathrm{C}$ & 1.368 & -1.417 & -0.112 & $\mathrm{C}$ & -1.356 & 1.409 & -0.115 \\
\hline $\mathrm{C}$ & -2.545 & -1.267 & -0.013 & $\mathrm{C}$ & -2.545 & -1.156 & -0.066 & $\mathrm{C}$ & 2.565 & 1.115 & -0.068 \\
\hline $\mathrm{C}$ & -3.125 & -0.007 & 0.089 & $\mathrm{C}$ & -3.130 & 0.086 & 0.009 & $\mathrm{C}$ & 3.140 & -0.119 & 0.013 \\
\hline $\mathrm{C}$ & -4.505 & 0.300 & 0.076 & $\mathrm{C}$ & -4.534 & 0.371 & 0.025 & $\mathrm{C}$ & 4.557 & -0.406 & 0.031 \\
\hline $\mathrm{C}$ & -4.914 & 1.666 & 0.162 & $\mathrm{C}$ & -4.963 & 1.725 & 0.069 & $\mathrm{C}$ & 4.982 & -1.756 & 0.081 \\
\hline $\mathrm{C}$ & -6.235 & 2.035 & 0.155 & $\mathrm{C}$ & -6.297 & 2.068 & 0.084 & $\mathrm{C}$ & 6.321 & -2.101 & 0.095 \\
\hline $\mathrm{C}$ & -7.259 & 1.048 & 0.042 & $\mathrm{C}$ & -7.294 & 1.060 & 0.046 & $\mathrm{C}$ & 7.310 & -1.094 & 0.052 \\
\hline $\mathrm{C}$ & -6.866 & -0.323 & -0.046 & $\mathrm{C}$ & -6.883 & -0.299 & 0.003 & $\mathrm{C}$ & 6.905 & 0.260 & 0.004 \\
\hline $\mathrm{C}$ & -5.543 & -0.678 & -0.020 & $\mathrm{C}$ & -5.547 & -0.628 & 0.000 & $\mathrm{C}$ & 5.565 & 0.590 & -0.001 \\
\hline $\mathrm{C}$ & -9.033 & 2.629 & 0.607 & $\mathrm{C}$ & -9.100 & 2.573 & 0.702 & $\mathrm{C}$ & 9.133 & -2.547 & 0.800 \\
\hline $\mathrm{C}$ & -8.635 & 2.986 & 1.909 & $\mathrm{C}$ & -8.620 & 2.917 & 1.983 & $\mathrm{C}$ & 8.625 & -2.808 & 2.092 \\
\hline $\mathrm{C}$ & -9.090 & 4.163 & 2.477 & $\mathrm{C}$ & -9.079 & 4.056 & 2.614 & $\mathrm{C}$ & 9.083 & -3.891 & 2.813 \\
\hline $\mathrm{C}$ & -9.959 & 5.011 & 1.761 & $\mathrm{C}$ & -10.031 & 4.888 & 1.986 & $\mathrm{C}$ & 10.062 & -4.751 & 2.269 \\
\hline $\mathrm{C}$ & -10.365 & 4.651 & 0.464 & $\mathrm{C}$ & -10.515 & 4.545 & 0.710 & $\mathrm{C}$ & 10.572 & -4.495 & 0.981 \\
\hline $\mathrm{C}$ & -9.905 & 3.466 & -0.102 & $\mathrm{C}$ & -10.053 & 3.396 & 0.080 & $\mathrm{C}$ & 10.111 & -3.402 & 0.261 \\
\hline $\mathrm{C}$ & -11.244 & 7.033 & 1.763 & $\mathrm{C}$ & -11.388 & 6.858 & 2.138 & $\mathrm{C}$ & 11.439 & -6.687 & 2.596 \\
\hline $\mathrm{C}$ & -9.569 & 0.562 & -0.587 & $\mathrm{C}$ & -9.601 & 0.560 & -0.590 & $\mathrm{C}$ & 9.604 & -0.658 & -0.678 \\
\hline $\mathrm{C}$ & -10.765 & 0.281 & 0.086 & $\mathrm{C}$ & -10.822 & 0.271 & 0.041 & $\mathrm{C}$ & 10.865 & -0.354 & -0.132 \\
\hline $\mathrm{C}$ & -11.738 & -0.518 & -0.505 & $\mathrm{C}$ & -11.770 & -0.530 & -0.584 & $\mathrm{C}$ & 11.785 & 0.397 & -0.850 \\
\hline $\mathrm{C}$ & -11.526 & -1.045 & -1.792 & $\mathrm{C}$ & -11.510 & -1.055 & -1.864 & $\mathrm{C}$ & 11.461 & 0.858 & -2.141 \\
\hline $\mathrm{C}$ & -10.326 & -0.752 & -2.471 & $\mathrm{C}$ & -10.287 & -0.758 & -2.501 & $\mathrm{C}$ & 10.197 & 0.550 & -2.691 \\
\hline $\mathrm{C}$ & -9.362 & 0.043 & -1.879 & $\mathrm{C}$ & -9.346 & 0.037 & -1.875 & $\mathrm{C}$ & 9.283 & -0.194 & -1.973 \\
\hline $\mathrm{C}$ & -13.651 & -2.149 & -1.853 & $\mathrm{C}$ & -13.627 & -2.171 & -2.002 & $\mathrm{C}$ & 13.581 & 1.931 & -2.458 \\
\hline $\mathrm{C}$ & -1.647 & -4.591 & 1.125 & $\mathrm{C}$ & -1.345 & -4.663 & 0.998 & $\mathrm{C}$ & 1.360 & 4.658 & 0.946 \\
\hline $\mathrm{C}$ & -2.476 & -5.841 & 0.871 & $\mathrm{C}$ & -2.234 & -5.874 & 0.756 & $\mathrm{C}$ & 2.267 & 5.853 & 0.692 \\
\hline $\mathrm{C}$ & -3.864 & -5.596 & 0.257 & $\mathrm{C}$ & -3.713 & -5.560 & 0.475 & $\mathrm{C}$ & 3.746 & 5.516 & 0.444 \\
\hline $\mathrm{C}$ & -4.836 & -4.840 & 1.170 & $\mathrm{C}$ & -4.448 & -4.875 & 1.633 & $\mathrm{C}$ & 4.454 & 4.849 & 1.630 \\
\hline $\mathrm{C}$ & 1.224 & -5.099 & -0.929 & $\mathrm{C}$ & 1.395 & -4.747 & -1.330 & $\mathrm{C}$ & -1.411 & 4.667 & -1.407 \\
\hline $\mathrm{C}$ & 2.489 & -5.859 & -1.297 & $\mathrm{C}$ & 2.604 & -5.666 & -1.398 & $\mathrm{C}$ & -2.592 & 5.624 & -1.452 \\
\hline $\mathrm{C}$ & 3.357 & -6.302 & -0.107 & $\mathrm{C}$ & 2.834 & -6.541 & -0.154 & $\mathrm{C}$ & -2.711 & 6.579 & -0.253 \\
\hline $\mathrm{C}$ & 2.690 & -7.325 & 0.819 & $\mathrm{C}$ & 1.766 & -7.618 & 0.068 & $\mathrm{C}$ & -1.603 & 7.635 & -0.174 \\
\hline $\mathrm{C}$ & 2.717 & -1.247 & -0.094 & $\mathrm{C}$ & 2.753 & -1.142 & -0.121 & $\mathrm{C}$ & -2.755 & 1.132 & -0.121 \\
\hline $\mathrm{C}$ & 3.299 & 0.017 & 0.000 & $\mathrm{C}$ & 3.335 & 0.104 & -0.059 & $\mathrm{C}$ & -3.341 & -0.098 & -0.059 \\
\hline $\mathrm{C}$ & 4.676 & 0.321 & -0.040 & $\mathrm{C}$ & 4.737 & 0.393 & -0.074 & $\mathrm{C}$ & -4.760 & -0.375 & -0.070 \\
\hline
\end{tabular}




\begin{tabular}{|c|c|c|c|c|c|c|c|c|c|c|c|}
\hline $\mathrm{C}$ & 5.093 & 1.683 & 0.089 & $\mathrm{C}$ & 5.163 & 1.747 & 0.011 & $\mathrm{C}$ & -5.197 & -1.721 & -0.005 \\
\hline $\mathrm{C}$ & 6.413 & 2.049 & 0.045 & $\mathrm{C}$ & 6.495 & 2.096 & -0.008 & $\mathrm{C}$ & -6.538 & -2.056 & -0.019 \\
\hline $\mathrm{C}$ & 7.432 & 1.062 & -0.113 & $\mathrm{C}$ & 7.495 & 1.094 & -0.101 & $\mathrm{C}$ & -7.519 & -1.043 & -0.091 \\
\hline $\mathrm{C}$ & 7.032 & -0.306 & -0.232 & $\mathrm{C}$ & 7.087 & -0.265 & -0.179 & $\mathrm{C}$ & -7.103 & 0.308 & -0.150 \\
\hline $\mathrm{C}$ & 5.709 & -0.655 & -0.207 & $\mathrm{C}$ & 5.753 & -0.598 & -0.173 & $\mathrm{C}$ & -5.760 & 0.627 & -0.144 \\
\hline $\mathrm{C}$ & 9.784 & 0.485 & 0.197 & $\mathrm{C}$ & 9.824 & 0.546 & 0.402 & $\mathrm{C}$ & -9.830 & -0.530 & 0.532 \\
\hline $\mathrm{C}$ & 10.902 & 0.321 & -0.643 & $\mathrm{C}$ & 11.020 & 0.315 & -0.310 & $\mathrm{C}$ & -11.070 & -0.255 & -0.086 \\
\hline $\mathrm{C}$ & 11.908 & -0.560 & -0.294 & $\mathrm{C}$ & 11.980 & -0.535 & 0.202 & $\mathrm{C}$ & -11.993 & 0.556 & 0.541 \\
\hline $\mathrm{C}$ & 11.830 & -1.294 & 0.908 & $\mathrm{C}$ & 11.781 & -1.173 & 1.445 & $\mathrm{C}$ & -11.714 & 1.113 & 1.808 \\
\hline $\mathrm{C}$ & 10.719 & -1.124 & 1.753 & $\mathrm{C}$ & 10.593 & -0.940 & 2.161 & $\mathrm{C}$ & -10.480 & 0.839 & 2.430 \\
\hline $\mathrm{C}$ & 9.708 & -0.238 & 1.396 & $\mathrm{C}$ & 9.627 & -0.087 & 1.640 & $\mathrm{C}$ & -9.552 & 0.026 & 1.793 \\
\hline $\mathrm{C}$ & 12.878 & -2.890 & 2.355 & $\mathrm{C}$ & 12.670 & -2.659 & 3.102 & $\mathrm{C}$ & -12.490 & 2.491 & 3.610 \\
\hline $\mathrm{C}$ & 9.164 & 2.724 & -0.560 & $\mathrm{C}$ & 9.275 & 2.672 & -0.668 & $\mathrm{C}$ & -9.334 & -2.539 & -0.769 \\
\hline $\mathrm{C}$ & 10.124 & 3.421 & 0.187 & $\mathrm{C}$ & 10.258 & 3.428 & -0.009 & $\mathrm{C}$ & -10.346 & -3.332 & -0.197 \\
\hline $\mathrm{C}$ & 10.547 & 4.685 & -0.209 & $\mathrm{C}$ & 10.702 & 4.632 & -0.541 & $\mathrm{C}$ & -10.801 & -4.473 & -0.841 \\
\hline $\mathrm{C}$ & 10.016 & 5.269 & -1.373 & $\mathrm{C}$ & 10.168 & 5.099 & -1.757 & $\mathrm{C}$ & -10.250 & -4.844 & -2.083 \\
\hline $\mathrm{C}$ & 9.058 & 4.562 & -2.129 & $\mathrm{C}$ & 9.185 & 4.336 & -2.423 & $\mathrm{C}$ & -9.237 & -4.048 & -2.660 \\
\hline $\mathrm{C}$ & 8.640 & 3.306 & -1.731 & $\mathrm{C}$ & 8.746 & 3.141 & -1.889 & $\mathrm{C}$ & -8.785 & -2.915 & -2.015 \\
\hline $\mathrm{C}$ & 11.341 & 7.254 & -1.162 & $\mathrm{C}$ & 11.534 & 7.069 & -1.775 & $\mathrm{C}$ & -11.654 & -6.776 & -2.301 \\
\hline $\mathrm{H}$ & -3.172 & -2.148 & -0.120 & $\mathrm{H}$ & -3.162 & -2.048 & -0.127 & $\mathrm{H}$ & 3.185 & 2.005 & -0.134 \\
\hline $\mathrm{H}$ & -2.457 & 0.848 & 0.186 & $\mathrm{H}$ & -2.479 & 0.958 & 0.056 & $\mathrm{H}$ & 2.491 & -0.992 & 0.066 \\
\hline $\mathrm{H}$ & -4.151 & 2.437 & 0.215 & $\mathrm{H}$ & -4.215 & 2.513 & 0.070 & $\mathrm{H}$ & 4.235 & -2.545 & 0.090 \\
\hline $\mathrm{H}$ & -6.507 & 3.082 & 0.198 & $\mathrm{H}$ & -6.590 & 3.112 & 0.091 & $\mathrm{H}$ & 6.614 & -3.144 & 0.106 \\
\hline $\mathrm{H}$ & -7.630 & -1.089 & -0.097 & $\mathrm{H}$ & -7.632 & -1.082 & 0.008 & $\mathrm{H}$ & 7.656 & 1.043 & 0.003 \\
\hline $\mathrm{H}$ & -5.291 & -1.733 & -0.053 & $\mathrm{H}$ & -5.273 & -1.678 & -0.001 & $\mathrm{H}$ & 5.292 & 1.640 & -0.012 \\
\hline $\mathrm{H}$ & -7.987 & 2.326 & 2.477 & $\mathrm{H}$ & -7.909 & 2.269 & 2.485 & $\mathrm{H}$ & 7.892 & -2.138 & 2.528 \\
\hline $\mathrm{H}$ & -8.807 & 4.446 & 3.485 & $\mathrm{H}$ & -8.736 & 4.325 & 3.607 & $\mathrm{H}$ & 8.719 & -4.094 & 3.814 \\
\hline $\mathrm{H}$ & -11.029 & 5.287 & -0.108 & $\mathrm{H}$ & -11.238 & 5.172 & 0.202 & $\mathrm{H}$ & 11.312 & -5.149 & 0.536 \\
\hline $\mathrm{H}$ & -10.215 & 3.195 & -1.106 & $\mathrm{H}$ & -10.416 & 3.144 & -0.910 & $\mathrm{H}$ & 10.490 & -3.221 & -0.740 \\
\hline $\mathrm{H}$ & -11.409 & 7.838 & 2.479 & $\mathrm{H}$ & -11.529 & 7.635 & 2.889 & $\mathrm{H}$ & 11.570 & -7.404 & 3.406 \\
\hline $\mathrm{H}$ & -12.198 & 6.544 & 1.535 & $\mathrm{H}$ & -12.336 & 6.338 & 1.964 & $\mathrm{H}$ & 12.384 & -6.168 & 2.406 \\
\hline $\mathrm{H}$ & -10.805 & 7.442 & 0.846 & $\mathrm{H}$ & -11.030 & 7.309 & 1.205 & $\mathrm{H}$ & 11.108 & -7.210 & 1.691 \\
\hline $\mathrm{H}$ & -10.929 & 0.679 & 1.082 & $\mathrm{H}$ & -11.019 & 0.661 & 1.034 & $\mathrm{H}$ & 11.110 & -0.687 & 0.870 \\
\hline $\mathrm{H}$ & -12.650 & -0.731 & 0.040 & $\mathrm{H}$ & -12.697 & -0.751 & -0.070 & $\mathrm{H}$ & 12.742 & 0.634 & -0.399 \\
\hline $\mathrm{H}$ & -10.191 & -1.147 & -3.473 & $\mathrm{H}$ & -10.115 & -1.152 & -3.497 & $\mathrm{H}$ & 9.975 & 0.896 & -3.695 \\
\hline $\mathrm{H}$ & -8.453 & 0.286 & -2.421 & $\mathrm{H}$ & -8.420 & 0.283 & -2.384 & $\mathrm{H}$ & 8.325 & -0.448 & -2.415 \\
\hline $\mathrm{H}$ & -14.174 & -2.770 & -2.581 & $\mathrm{H}$ & -14.118 & -2.792 & -2.751 & $\mathrm{H}$ & 14.035 & 2.502 & -3.268 \\
\hline $\mathrm{H}$ & -14.237 & -1.245 & -1.653 & $\mathrm{H}$ & -14.225 & -1.270 & -1.821 & $\mathrm{H}$ & 14.176 & 1.032 & -2.263 \\
\hline $\mathrm{H}$ & -13.507 & -2.715 & -0.925 & $\mathrm{H}$ & -13.512 & -2.738 & -1.072 & $\mathrm{H}$ & 13.527 & 2.550 & -1.556 \\
\hline $\mathrm{H}$ & -2.161 & -3.877 & 1.779 & $\mathrm{H}$ & -1.691 & -4.062 & 1.847 & $\mathrm{H}$ & 1.685 & 4.072 & 1.814 \\
\hline $\mathrm{H}$ & -0.686 & -4.845 & 1.588 & $\mathrm{H}$ & -0.310 & -4.967 & 1.191 & $\mathrm{H}$ & 0.326 & 4.978 & 1.115 \\
\hline $\mathrm{H}$ & -1.901 & -6.511 & 0.220 & $\mathrm{H}$ & -1.818 & -6.451 & -0.080 & $\mathrm{H}$ & 1.873 & 6.414 & -0.165 \\
\hline $\mathrm{H}$ & -2.580 & -6.358 & 1.835 & $\mathrm{H}$ & -2.151 & -6.512 & 1.647 & $\mathrm{H}$ & 2.177 & 6.513 & 1.565 \\
\hline $\mathrm{H}$ & -4.298 & -6.571 & 0.007 & $\mathrm{H}$ & -4.217 & -6.506 & 0.241 & $\mathrm{H}$ & 4.267 & 6.449 & 0.197 \\
\hline $\mathrm{H}$ & -3.747 & -5.063 & -0.695 & $\mathrm{H}$ & -3.783 & -4.946 & -0.432 & $\mathrm{H}$ & 3.824 & 4.879 & -0.446 \\
\hline $\mathrm{H}$ & -5.818 & -4.739 & 0.694 & $\mathrm{H}$ & -5.510 & -4.743 & 1.395 & $\mathrm{H}$ & 5.518 & 4.699 & 1.414 \\
\hline $\mathrm{H}$ & -4.482 & -3.830 & 1.414 & $\mathrm{H}$ & -4.036 & -3.884 & 1.859 & $\mathrm{H}$ & 4.025 & 3.869 & 1.872 \\
\hline $\mathrm{H}$ & -4.985 & -5.369 & 2.119 & $\mathrm{H}$ & -4.391 & -5.472 & 2.551 & $\mathrm{H}$ & 4.388 & 5.468 & 2.533 \\
\hline $\mathrm{H}$ & 0.637 & -4.840 & -1.815 & $\mathrm{H}$ & 1.279 & -4.179 & -2.261 & $\mathrm{H}$ & -1.379 & 4.043 & -2.308 \\
\hline $\mathrm{H}$ & 0.592 & -5.668 & -0.244 & $\mathrm{H}$ & 0.467 & -5.292 & -1.143 & $\mathrm{H}$ & -0.454 & 5.188 & -1.317 \\
\hline
\end{tabular}




\begin{tabular}{|c|c|c|c|c|c|c|c|c|c|c|c|}
\hline $\mathrm{H}$ & 3.079 & -5.238 & -1.983 & $\mathrm{H}$ & 3.492 & -5.050 & -1.589 & $\mathrm{H}$ & -3.510 & 5.029 & -1.545 \\
\hline $\mathrm{H}$ & 2.171 & -6.740 & -1.871 & $\mathrm{H}$ & 2.472 & -6.305 & -2.282 & $\mathrm{H}$ & -2.502 & 6.204 & -2.381 \\
\hline $\mathrm{H}$ & 4.282 & -6.733 & -0.509 & $\mathrm{H}$ & 3.812 & -7.027 & -0.259 & $\mathrm{H}$ & -3.681 & 7.088 & -0.320 \\
\hline $\mathrm{H}$ & 3.657 & -5.418 & 0.471 & $\mathrm{H}$ & 2.907 & -5.897 & 0.732 & $\mathrm{H}$ & -2.738 & 5.992 & 0.674 \\
\hline $\mathrm{H}$ & 3.381 & -7.643 & 1.607 & $\mathrm{H}$ & 2.006 & -8.230 & 0.944 & $\mathrm{H}$ & -1.762 & 8.305 & 0.678 \\
\hline $\mathrm{H}$ & 2.384 & -8.221 & 0.267 & $\mathrm{H}$ & 1.695 & -8.290 & -0.796 & $\mathrm{H}$ & -1.577 & 8.252 & -1.080 \\
\hline $\mathrm{H}$ & 1.801 & -6.918 & 1.315 & $\mathrm{H}$ & 0.771 & -7.189 & 0.236 & $\mathrm{H}$ & -0.610 & 7.186 & -0.052 \\
\hline $\mathrm{H}$ & 3.345 & -2.126 & -0.199 & $\mathrm{H}$ & 3.373 & -2.033 & -0.182 & $\mathrm{H}$ & -3.368 & 2.029 & -0.178 \\
\hline $\mathrm{H}$ & 2.633 & 0.872 & 0.114 & $\mathrm{H}$ & 2.682 & 0.972 & 0.009 & $\mathrm{H}$ & -2.700 & -0.977 & 0.001 \\
\hline $\mathrm{H}$ & 4.337 & 2.448 & 0.243 & $\mathrm{H}$ & 4.414 & 2.528 & 0.110 & $\mathrm{H}$ & -4.456 & -2.513 & 0.072 \\
\hline $\mathrm{H}$ & 6.693 & 3.089 & 0.166 & $\mathrm{H}$ & 6.786 & 3.136 & 0.082 & $\mathrm{H}$ & -6.841 & -3.094 & 0.054 \\
\hline $\mathrm{H}$ & 7.790 & -1.065 & -0.384 & $\mathrm{H}$ & 7.838 & -1.040 & -0.282 & $\mathrm{H}$ & -7.846 & 1.093 & -0.233 \\
\hline $\mathrm{H}$ & 5.447 & -1.700 & -0.341 & $\mathrm{H}$ & 5.481 & -1.644 & -0.267 & $\mathrm{H}$ & -5.477 & 1.672 & -0.215 \\
\hline $\mathrm{H}$ & 10.965 & 0.879 & -1.571 & $\mathrm{H}$ & 11.174 & 0.793 & -1.272 & $\mathrm{H}$ & -11.284 & -0.663 & -1.068 \\
\hline $\mathrm{H}$ & 12.770 & -0.709 & -0.934 & $\mathrm{H}$ & 12.898 & -0.736 & -0.340 & $\mathrm{H}$ & -12.942 & 0.793 & 0.072 \\
\hline $\mathrm{H}$ & 10.648 & -1.659 & 2.693 & $\mathrm{H}$ & 10.426 & -1.401 & 3.127 & $\mathrm{H}$ & -10.251 & 1.238 & 3.410 \\
\hline $\mathrm{H}$ & 8.865 & -0.088 & 2.064 & $\mathrm{H}$ & 8.724 & 0.112 & 2.209 & $\mathrm{H}$ & -8.614 & -0.205 & 2.288 \\
\hline $\mathrm{H}$ & 13.803 & -3.466 & 2.322 & $\mathrm{H}$ & 13.589 & -3.235 & 3.200 & $\mathrm{H}$ & -13.402 & 3.056 & 3.802 \\
\hline $\mathrm{H}$ & 12.021 & -3.573 & 2.393 & $\mathrm{H}$ & 11.808 & -3.337 & 3.103 & $\mathrm{H}$ & -11.631 & 3.171 & 3.595 \\
\hline $\mathrm{H}$ & 12.880 & -2.244 & 3.240 & $\mathrm{H}$ & 12.590 & -1.950 & 3.933 & $\mathrm{H}$ & -12.356 & 1.733 & 4.389 \\
\hline $\mathrm{H}$ & 10.531 & 2.976 & 1.089 & $\mathrm{H}$ & 10.662 & 3.078 & 0.936 & $\mathrm{H}$ & -10.758 & -3.061 & 0.770 \\
\hline $\mathrm{H}$ & 11.282 & 5.208 & 0.391 & $\mathrm{H}$ & 11.450 & 5.204 & -0.006 & $\mathrm{H}$ & -11.569 & -5.076 & -0.371 \\
\hline $\mathrm{H}$ & 8.678 & 5.019 & -3.037 & $\mathrm{H}$ & 8.803 & 4.701 & -3.370 & $\mathrm{H}$ & -8.841 & -4.339 & -3.627 \\
\hline $\mathrm{H}$ & 7.924 & 2.757 & -2.334 & $\mathrm{H}$ & 8.011 & 2.546 & -2.422 & $\mathrm{H}$ & -8.026 & -2.294 & -2.479 \\
\hline $\mathrm{H}$ & 11.447 & 8.171 & -1.741 & $\mathrm{H}$ & 11.649 & 7.914 & -2.453 & $\mathrm{H}$ & -11.770 & -7.555 & -3.054 \\
\hline $\mathrm{H}$ & 12.301 & 6.727 & -1.128 & $\mathrm{H}$ & 12.483 & 6.529 & -1.695 & $\mathrm{H}$ & -12.594 & -6.225 & -2.192 \\
\hline $\mathrm{H}$ & 11.011 & 7.498 & -0.145 & $\mathrm{H}$ & 11.221 & 7.428 & -0.789 & $\mathrm{H}$ & -11.369 & -7.228 & -1.345 \\
\hline
\end{tabular}


Table S11. XYZ coordinates and energies (hr) for for the singlet, broken symmetry (BS), and triplet states of the Chichibabin hydrocarbon.

\begin{tabular}{|c|c|c|c|c|c|c|c|c|c|c|c|}
\hline \multicolumn{4}{|c|}{$\begin{array}{l}\text { singlet } \\
(-1464.87576091 \mathrm{hr})\end{array}$} & \multicolumn{4}{|c|}{$\begin{array}{l}\text { BS } \\
(-1464.88095919 \mathrm{hr})\end{array}$} & \multicolumn{4}{|c|}{$\begin{array}{l}\text { triplet } \\
(-1464.87753662 \mathrm{hr})\end{array}$} \\
\hline $\mathrm{C}$ & -2.848 & -1.213 & -0.096 & $\mathrm{C}$ & 0.252 & 1.182 & 2.858 & $\mathrm{C}$ & 1.149 & 0.361 & 2.862 \\
\hline $\mathrm{C}$ & -1.483 & -1.207 & -0.104 & $\mathrm{C}$ & 0.255 & 1.178 & 1.477 & $\mathrm{C}$ & 1.147 & 0.357 & 1.473 \\
\hline $\mathrm{C}$ & -0.710 & 0.000 & 0.000 & $\mathrm{C}$ & 0.001 & 0.002 & 0.731 & $\mathrm{C}$ & 0.000 & 0.000 & 0.740 \\
\hline $\mathrm{C}$ & -1.483 & 1.207 & 0.103 & $\mathrm{C}$ & -0.253 & -1.175 & 1.477 & $\mathrm{C}$ & -1.147 & -0.357 & 1.473 \\
\hline $\mathrm{C}$ & -2.848 & 1.213 & 0.095 & $\mathrm{C}$ & -0.249 & -1.179 & 2.859 & $\mathrm{C}$ & -1.149 & -0.361 & 2.862 \\
\hline $\mathrm{C}$ & -3.628 & 0.000 & 0.000 & $\mathrm{C}$ & 0.002 & 0.002 & 3.615 & $\mathrm{C}$ & 0.000 & 0.000 & 3.606 \\
\hline $\mathrm{C}$ & 0.710 & 0.000 & 0.000 & $\mathrm{C}$ & 0.000 & 0.001 & -0.732 & $\mathrm{C}$ & 0.000 & 0.000 & -0.740 \\
\hline $\mathrm{C}$ & 1.483 & -1.207 & 0.103 & $\mathrm{C}$ & -0.251 & 1.178 & -1.478 & $\mathrm{C}$ & 1.147 & -0.357 & -1.473 \\
\hline $\mathrm{C}$ & 2.848 & -1.213 & 0.095 & $\mathrm{C}$ & -0.248 & 1.183 & -2.859 & $\mathrm{C}$ & 1.149 & -0.361 & -2.862 \\
\hline $\mathrm{C}$ & 3.628 & 0.000 & 0.000 & $\mathrm{C}$ & 0.000 & 0.001 & -3.616 & $\mathrm{C}$ & 0.000 & 0.000 & -3.606 \\
\hline $\mathrm{C}$ & 2.848 & 1.213 & -0.095 & $\mathrm{C}$ & 0.248 & -1.180 & -2.859 & $\mathrm{C}$ & -1.149 & 0.361 & -2.862 \\
\hline $\mathrm{C}$ & 1.483 & 1.207 & -0.104 & $\mathrm{C}$ & 0.252 & -1.175 & -1.478 & $\mathrm{C}$ & -1.147 & 0.357 & -1.473 \\
\hline $\mathrm{C}$ & -5.030 & 0.000 & 0.000 & $\mathrm{C}$ & 0.002 & 0.002 & 5.057 & $\mathrm{C}$ & 0.000 & 0.000 & 5.069 \\
\hline $\mathrm{C}$ & 5.030 & 0.000 & 0.000 & $\mathrm{C}$ & -0.001 & 0.001 & -5.057 & $\mathrm{C}$ & 0.000 & 0.000 & -5.069 \\
\hline $\mathrm{C}$ & -5.807 & -1.230 & 0.268 & $\mathrm{C}$ & -0.293 & 1.231 & 5.809 & $\mathrm{C}$ & 0.799 & 0.985 & 5.801 \\
\hline $\mathrm{C}$ & -5.456 & -2.115 & 1.309 & $\mathrm{C}$ & -1.277 & 2.147 & 5.374 & $\mathrm{C}$ & 0.992 & 2.297 & 5.303 \\
\hline $\mathrm{C}$ & -6.205 & -3.262 & 1.563 & $\mathrm{C}$ & -1.557 & 3.304 & 6.096 & $\mathrm{C}$ & 1.749 & 3.231 & 6.002 \\
\hline $\mathrm{C}$ & -7.326 & -3.558 & 0.786 & $\mathrm{C}$ & -0.862 & 3.587 & 7.275 & $\mathrm{C}$ & 2.346 & 2.892 & 7.219 \\
\hline $\mathrm{C}$ & -7.696 & -2.689 & -0.244 & $\mathrm{C}$ & 0.113 & 2.693 & 7.726 & $\mathrm{C}$ & 2.173 & 1.602 & 7.730 \\
\hline $\mathrm{C}$ & -6.953 & -1.539 & -0.494 & $\mathrm{C}$ & 0.390 & 1.532 & 7.010 & $\mathrm{C}$ & 1.411 & 0.666 & 7.037 \\
\hline $\mathrm{C}$ & -5.807 & 1.230 & -0.268 & $\mathrm{C}$ & 0.297 & -1.228 & 5.809 & $\mathrm{C}$ & -0.799 & -0.985 & 5.801 \\
\hline $\mathrm{C}$ & -5.456 & 2.115 & -1.309 & $\mathrm{C}$ & 1.281 & -2.144 & 5.373 & $\mathrm{C}$ & -0.992 & -2.297 & 5.303 \\
\hline $\mathrm{C}$ & -6.205 & 3.262 & -1.563 & $\mathrm{C}$ & 1.561 & -3.301 & 6.095 & $\mathrm{C}$ & -1.749 & -3.231 & 6.002 \\
\hline $\mathrm{C}$ & -7.327 & 3.558 & -0.785 & $\mathrm{C}$ & 0.867 & -3.583 & 7.274 & $\mathrm{C}$ & -2.346 & -2.892 & 7.219 \\
\hline $\mathrm{C}$ & -7.697 & 2.689 & 0.245 & $\mathrm{C}$ & -0.108 & -2.690 & 7.726 & $\mathrm{C}$ & -2.173 & -1.602 & 7.730 \\
\hline $\mathrm{C}$ & -6.954 & 1.538 & 0.494 & $\mathrm{C}$ & -0.386 & -1.529 & 7.010 & $\mathrm{C}$ & -1.411 & -0.666 & 7.037 \\
\hline $\mathrm{C}$ & 5.807 & 1.230 & 0.268 & $\mathrm{C}$ & -0.298 & -1.227 & -5.809 & $\mathrm{C}$ & -0.799 & 0.985 & -5.801 \\
\hline $\mathrm{C}$ & 6.953 & 1.538 & -0.494 & $\mathrm{C}$ & 0.383 & -1.530 & -7.010 & $\mathrm{C}$ & -1.411 & 0.666 & -7.037 \\
\hline $\mathrm{C}$ & 7.697 & 2.689 & -0.244 & $\mathrm{C}$ & 0.103 & -2.690 & -7.727 & $\mathrm{C}$ & -2.173 & 1.602 & -7.730 \\
\hline $\mathrm{C}$ & 7.327 & 3.558 & 0.786 & $\mathrm{C}$ & -0.874 & -3.582 & -7.275 & $\mathrm{C}$ & -2.346 & 2.892 & -7.219 \\
\hline $\mathrm{C}$ & 6.205 & 3.262 & 1.563 & $\mathrm{C}$ & -1.567 & -3.297 & -6.096 & $\mathrm{C}$ & -1.749 & 3.231 & -6.002 \\
\hline $\mathrm{C}$ & 5.456 & 2.115 & 1.309 & $\mathrm{C}$ & -1.285 & -2.141 & -5.373 & $\mathrm{C}$ & -0.992 & 2.297 & -5.303 \\
\hline $\mathrm{C}$ & 5.807 & -1.230 & -0.268 & $\mathrm{C}$ & 0.297 & 1.230 & -5.810 & $\mathrm{C}$ & 0.799 & -0.985 & -5.801 \\
\hline $\mathrm{C}$ & 6.953 & -1.538 & 0.494 & $\mathrm{C}$ & -0.386 & 1.533 & -7.010 & $\mathrm{C}$ & 1.411 & -0.666 & -7.037 \\
\hline $\mathrm{C}$ & 7.697 & -2.689 & 0.244 & $\mathrm{C}$ & -0.106 & 2.693 & -7.726 & $\mathrm{C}$ & 2.173 & -1.602 & -7.730 \\
\hline $\mathrm{C}$ & 7.327 & -3.558 & -0.785 & $\mathrm{C}$ & 0.871 & 3.585 & -7.275 & $\mathrm{C}$ & 2.346 & -2.892 & -7.219 \\
\hline $\mathrm{C}$ & 6.205 & -3.262 & -1.563 & $\mathrm{C}$ & 1.565 & 3.300 & -6.097 & $\mathrm{C}$ & 1.749 & -3.231 & -6.002 \\
\hline $\mathrm{C}$ & 5.456 & -2.115 & -1.309 & $\mathrm{C}$ & 1.283 & 2.144 & -5.374 & $\mathrm{C}$ & 0.992 & -2.297 & -5.303 \\
\hline $\mathrm{H}$ & -3.368 & -2.155 & -0.224 & $\mathrm{H}$ & 0.493 & 2.102 & 3.382 & $\mathrm{H}$ & 2.056 & 0.634 & 3.390 \\
\hline $\mathrm{H}$ & -0.974 & -2.155 & -0.242 & $\mathrm{H}$ & 0.508 & 2.096 & 0.956 & $\mathrm{H}$ & 2.049 & 0.654 & 0.945 \\
\hline $\mathrm{H}$ & -0.974 & 2.155 & 0.241 & $\mathrm{H}$ & -0.506 & -2.093 & 0.956 & $\mathrm{H}$ & -2.049 & -0.654 & 0.945 \\
\hline $\mathrm{H}$ & -3.368 & 2.155 & 0.223 & $\mathrm{H}$ & -0.489 & -2.098 & 3.382 & $\mathrm{H}$ & -2.056 & -0.634 & 3.390 \\
\hline $\mathrm{H}$ & 0.974 & -2.155 & 0.242 & $\mathrm{H}$ & -0.502 & 2.097 & -0.957 & $\mathrm{H}$ & 2.049 & -0.654 & -0.945 \\
\hline $\mathrm{H}$ & 3.368 & -2.155 & 0.224 & $\mathrm{H}$ & -0.487 & 2.103 & -3.382 & $\mathrm{H}$ & 2.056 & -0.634 & -3.390 \\
\hline $\mathrm{H}$ & 3.368 & 2.155 & -0.224 & $\mathrm{H}$ & 0.486 & -2.100 & -3.383 & $\mathrm{H}$ & -2.056 & 0.634 & -3.390 \\
\hline $\mathrm{H}$ & 0.974 & 2.155 & -0.242 & $\mathrm{H}$ & 0.502 & -2.094 & -0.957 & $\mathrm{H}$ & -2.049 & 0.654 & -0.945 \\
\hline
\end{tabular}




\begin{tabular}{llll:llll:llll:}
$\mathrm{H}$ & -4.601 & -1.880 & 1.936 & $\mathrm{H}$ & -1.840 & 1.928 & 4.471 & $\mathrm{H}$ & 0.518 & 2.581 & 4.369 \\
$\mathrm{H}$ & -5.918 & -3.920 & 2.379 & $\mathrm{H}$ & -2.328 & 3.983 & 5.742 & $\mathrm{H}$ & 1.866 & 4.234 & 5.599 \\
$\mathrm{H}$ & -7.910 & -4.453 & 0.984 & $\mathrm{H}$ & -1.080 & 4.491 & 7.837 & $\mathrm{H}$ & 2.940 & 3.622 & 7.763 \\
$\mathrm{H}$ & -8.567 & -2.910 & -0.856 & $\mathrm{H}$ & 0.665 & 2.904 & 8.638 & $\mathrm{H}$ & 2.641 & 1.321 & 8.670 \\
$\mathrm{H}$ & -7.248 & -0.871 & -1.299 & $\mathrm{H}$ & 1.158 & 0.852 & 7.365 & $\mathrm{H}$ & 1.300 & -0.337 & 7.438 \\
$\mathrm{H}$ & -4.601 & 1.880 & -1.936 & $\mathrm{H}$ & 1.843 & -1.925 & 4.471 & $\mathrm{H}$ & -0.518 & -2.581 & 4.369 \\
$\mathrm{H}$ & -5.918 & 3.921 & -2.378 & $\mathrm{H}$ & 2.333 & -3.980 & 5.741 & $\mathrm{H}$ & -1.866 & -4.234 & 5.599 \\
$\mathrm{H}$ & -7.911 & 4.453 & -0.983 & $\mathrm{H}$ & 1.085 & -4.487 & 7.836 & $\mathrm{H}$ & -2.940 & -3.622 & 7.763 \\
$\mathrm{H}$ & -8.567 & 2.909 & 0.857 & $\mathrm{H}$ & -0.660 & -2.901 & 8.638 & $\mathrm{H}$ & -2.641 & -1.321 & 8.670 \\
$\mathrm{H}$ & -7.248 & 0.870 & 1.299 & $\mathrm{H}$ & -1.153 & -0.849 & 7.365 & $\mathrm{H}$ & -1.300 & 0.337 & 7.438 \\
$\mathrm{H}$ & 7.248 & 0.871 & -1.298 & $\mathrm{H}$ & 1.152 & -0.851 & -7.366 & $\mathrm{H}$ & -1.300 & -0.337 & -7.438 \\
$\mathrm{H}$ & 8.567 & 2.909 & -0.856 & $\mathrm{H}$ & 0.654 & -2.903 & -8.639 & $\mathrm{H}$ & -2.641 & 1.321 & -8.670 \\
$\mathrm{H}$ & 7.910 & 4.453 & 0.984 & $\mathrm{H}$ & -1.094 & -4.485 & -7.837 & $\mathrm{H}$ & -2.940 & 3.622 & -7.763 \\
$\mathrm{H}$ & 5.918 & 3.921 & 2.379 & $\mathrm{H}$ & -2.340 & -3.975 & -5.742 & $\mathrm{H}$ & -1.866 & 4.234 & -5.599 \\
$\mathrm{H}$ & 4.601 & 1.880 & 1.936 & $\mathrm{H}$ & -1.846 & -1.921 & -4.471 & $\mathrm{H}$ & -0.518 & 2.581 & -4.369 \\
$\mathrm{H}$ & 7.248 & -0.871 & 1.299 & $\mathrm{H}$ & -1.155 & 0.854 & -7.365 & $\mathrm{H}$ & 1.300 & 0.337 & -7.438 \\
$\mathrm{H}$ & 8.567 & -2.909 & 0.857 & $\mathrm{H}$ & -0.658 & 2.906 & -8.639 & $\mathrm{H}$ & 2.641 & -1.321 & -8.670 \\
$\mathrm{H}$ & 7.911 & -4.453 & -0.984 & $\mathrm{H}$ & 1.091 & 4.488 & -7.837 & $\mathrm{H}$ & 2.940 & -3.622 & -7.763 \\
$\mathrm{H}$ & 5.918 & -3.921 & -2.379 & $\mathrm{H}$ & 2.339 & 3.978 & -5.743 & $\mathrm{H}$ & 1.866 & -4.234 & -5.599 \\
$\mathrm{H}$ & 4.601 & -1.880 & -1.936 & $\mathrm{H}$ & 1.845 & 1.924 & -4.472 & $\mathrm{H}$ & 0.518 & -2.581 & -4.369 \\
\hline \hline
\end{tabular}

